\title{
SEARCHING FOR SPECTROSCOPIC BINARIES WITHIN TRANSITION DISK OBJECTS*
}

\author{
Saul A. Kohn ${ }^{1,2}$, Evgenya L. Shkolnik ${ }^{1,3}$, Alycia J. Weinberger ${ }^{4}$, Joleen K. Carlberg ${ }^{5}$, and Joe Llama ${ }^{1,6}$ \\ Lowell Observatory, 1400 W. Mars Hill Road, Flagstaff, AZ 86001, USA; saulkohn@sas.upenn.edu \\ ${ }^{2}$ Department of Physics and Astronomy, University of Pennsylvania, Philadelphia, PA, 19104, USA \\ ${ }^{3}$ School of Earth and Space Exploration, Arizona State University, Tempe, AZ 85287, USA \\ ${ }^{4}$ Department of Terrestrial Magnetism, Carnegie Institution of Washington, 5241 Broad Branch Road, NW, Washington, DC 20015, USA \\ 5 NASA/GSFC Code 667, Greenbelt, MD 20771, USA \\ ${ }^{6}$ SUPA, School of Physics \& Astronomy, North Haugh. St Andrews. Fife. KY16 9SS, UK \\ Received 2015 April 8; accepted 2016 January 28; published 2016 March 10
}

\begin{abstract}
Transition disks (TDs) are intermediate stage circumstellar disks characterized by an inner gap within the disk structure. To test whether these gaps may have been formed by closely orbiting, previously undetected stellar companions, we collected high-resolution optical spectra of $31 \mathrm{TD}$ objects to search for spectroscopic binaries (SBs). Twenty-four of these objects are in Ophiuchus and seven are within the Coronet, Corona Australis, and Chameleon I star-forming regions. We measured radial velocities for multiple epochs, obtaining a median precision of $400 \mathrm{~ms}^{-1}$. We identified double-lined SB SSTc2d J163154.7-250324 in Ophiuchus, which we determined to be composed of a $\mathrm{K} 7( \pm 0.5)$ and a $\mathrm{K} 9( \pm 0.5)$ star, with orbital limits of $a<0.6$ au and $P<150$ days. This results in an SB fraction of $0.04_{-0.03}^{+0.12}$ in Ophiuchus, which is consistent with other spectroscopic surveys of non-TD objects in the region. This similarity suggests that TDs are not preferentially sculpted by the presence of close binaries and that planet formation around close binaries may take place over similar timescales to that around single stars.
\end{abstract}

Key words: binaries: spectroscopic - circumstellar matter - stars: pre-main sequence

\section{INTRODUCTION}

Transition disks (TDs) are an intermediate stage of circumstellar disk evolution among pre-main sequence (PMS) stars. They are characterized by a lack of near-infrared excesses caused by inner optically thin holes or "gaps" opening within the disk structure, which typically have radii between one and tens of au and large far-infrared excesses from the disk material at larger separations (e.g., Hughes et al. 2007, 2009; Andrews et al. 2011; Zhu et al. 2011).

Planet formation and dust aggregation are thought to cause the gaps observed in TDs, with inner holes greater than 15 au possibly due to the formation of multiple planets (DodsonRobinson \& Salyk 2011; Zhu et al. 2011). Other gap-formation mechanisms may involve ultraviolet (UV) and X-ray photons irradiating the disk and accretion from the disk onto the host star (e.g., Gorti et al. 2009; Espaillat et al. 2012). Recent observations by Follette et al. (2013) support the results of simulations from, e.g., Rice et al. (2006) and Zhu \& Stone (2014) that dust dynamics may play a role in gap formation. The distribution of large and small grains in the inner regions of disks can be modified by gas pressure gradients, perhaps also generated by planets, that may trap or filter large grains as well as allow grains to grow efficiently (e.g., Zhu et al. 2011; Pinilla et al. 2012).

A closely orbiting stellar companion may also produce an inner gap in a circumstellar disk (Artymowicz \& Lubow 1994; D'Alessio et al. 2005), as illustrated by the CoKu Tauri/4 system. This system was observed to have a 10 au wide gap (Forrest et al. 2004). The initial interpretation for such a wide gap was that a $10 M_{\text {Jup }}$ planet orbiting the central star cleared out the material (Quillen et al. 2004). However, it was afterward shown that $\mathrm{CoKu}$ Tauri/4 was in fact a close visual binary (VB) system with a separation of $\sim 8$ au (Ireland \&

\footnotetext{
* This paper is based on data gathered with the $6.5 \mathrm{~m}$ Clay Telescope located at Las Campanas Observatory, Chile.
}

Kraus 2008), and that the secondary star likely carved out the gap. Similarly, Biller et al. (2012) also detected a VB within the TD object HD 142527. With a projected separation of $(12.8 \pm 1.5 \mathrm{au})$, the companion is likely to have played a role in shaping the system's complex disk structure, and it may have been responsible for the disk's cleared inner ring.

A close binary system need not preclude circumbinary planet formation. Using photometric data from Kepler, Doyle et al. (2011) announced the first discovery of a circumbinary planet. Several other circumbinary planets have since been discovered (e.g., Orosz et al. 2012; Qian et al. 2012, 2012b; Schwamb et al. 2013). These examples illustrate the need to further study multiple-star systems that host circumbinary material, and to test the effects of companion stars on disk dissipation and planet formation. Young star-forming regions (SFRs) are good test beds for such a study.

The nearby $(140 \mathrm{pc})$ Ophiuchus star-forming region (Oph) SFR contains a population of young stars of an average age 2.2 Myr (Wilking et al. 2005; Lombardi et al. 2008; Erickson et al. 2011). More than 50\% of the young stellar objects (YSOs) in Oph show evidence of optically thick circumstellar disks at mid-IR wavelengths (Bontemps et al. 2001), of which 9\% are identified as TDs (Evans et al. 2003; Cieza et al. 2010). This makes it well-suited for studies of disk dissipation.

Previous surveys of multiplicity in Oph and other SFRs have concentrated on visual binarity across all of the known active star-forming regions (e.g., Table 6 of Lafreniere et al. 2008). With the exception of interferometric methods that are sensitive to separations of order 0.1-1 au (Pott et al. 2010), VB searches are typically limited to separations $<4$ au (Ghez et al. 1993; Kraus et al. 2012). Cieza et al. (2010) (hereafter C10) used IR and optical data to identify the TDs in Oph. C10 also subclassify the TDs in their sample into five catagories: grain growth-dominated disks (which are accreting and have a negative slope of IR excess: $44 \%$ of their sample), giant planet- 


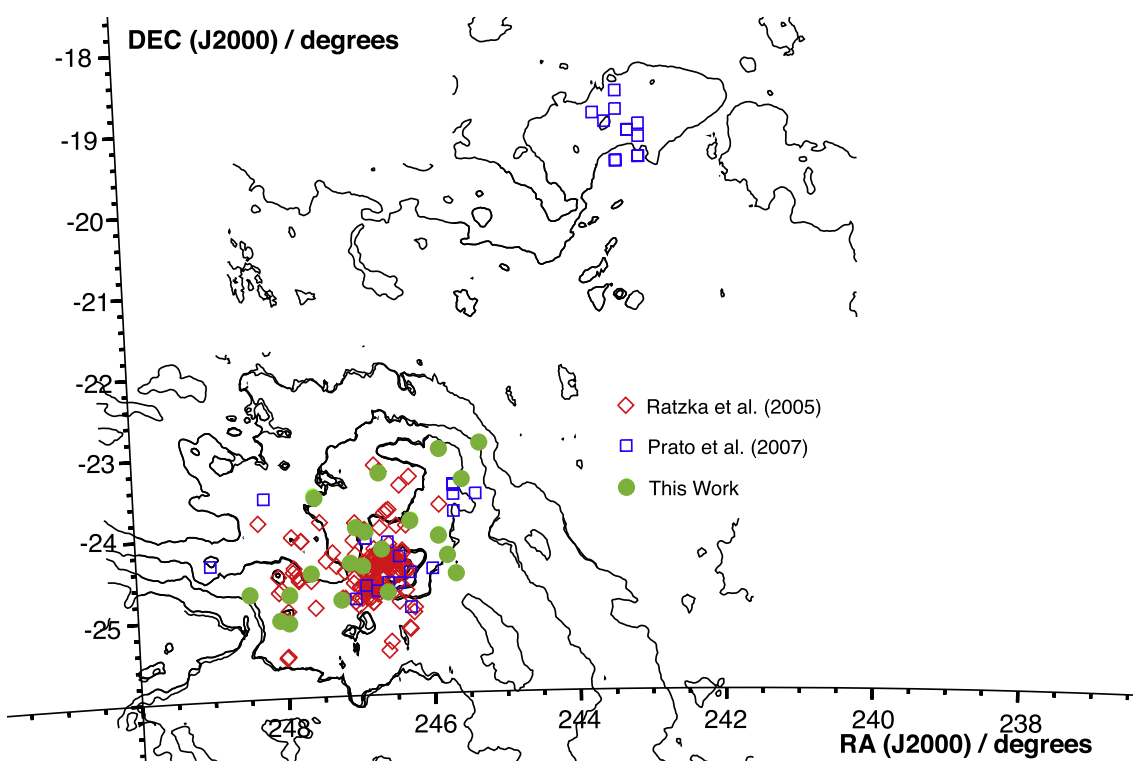

Figure 1. R.A. and decl. of our targets within Ophiuchus, overlaid with other multiplicity surveys. Contours are from the Infrared Astronomical Satellite (IRAS) Sky Survey Atlas at $100 \mu \mathrm{m}$ (Neugebauer et al. 1984; Beichman et al. 1988).

forming disks (accreting with a positive slope of IR excess: $13 \%$ ), photoevaporating disks (non-accreting with high disk luminosity: 17\%), disks already in the debris disk stage (nonaccreting with low disk luminosity: 13\%) and circumbinary disks $(13 \%)$. C10 did not observe an increase in detected companions within the separations to which their survey was most sensitive (8-20 au) compared with other separations, leading them to suggest that stellar companions at these separations are not responsible for a large fraction of the TD population.

We present a complementary radial velocity (RV) survey to $\mathrm{C} 10$ to search for stellar companions closer in to the primary star. Our aim is to test whether the spectroscopic binary (SB) fraction is similar or enhanced in the TD population relative to stars without such disks. The structure of this paper is as follows. The selection of the TD systems, observations and data reduction are described in Section 2. RV, rotational velocity, and spectral type (SpT) measurements are presented in Section 3. We report the discovery of a double-lined spectroscopic binary in Section 4 and evaluate possible biases and the completeness of our survey in Section 5. We derive a multiplicity fraction for Oph TDs, compare with previous multiplicity surveys of Oph, and discuss what multiplicity among TD objects might mean for theories of disk dispersal in Section 6. We summarize and conclude our study in Section 7. While not the main objective of this paper, we provide values of lithium I ( $\mathrm{Li}$ I) and $\mathrm{H} \alpha$ equivalent widths (EWs), accretion rates for our sample, and a discussion on the unusual nature of Oph star SR21A in the Appendix.

\section{SAMPLE AND OBSERVATIONS}

To survey the innermost regions of TD objects for stellar companions, we collected multi-epoch, high-resolution optical spectroscopy of 24 stars in Oph identified as having TDs (C10; Andrews \& Williams 2007a; Geers et al. 2007; Furlan et al. 2009), two in Corona Australis (CrA; <3 Myr; Neuhauser \& Forbrich 2008; Sicilia-Aguilar et al. 2008; Hughes et al. 2010), two in Coronet (Cor, embedded within CrA; $<1 \mathrm{Myr}$; Knacke et al. 1973; Sicilia-Aguilar et al. 2008; Hughes et al. 2010), and three in Chameleon I (Cha; 1-3 Myr; Furlan et al. 2009; Kim et al. 2009).

C10 identified Oph TDs from their colors as measured by the Spitzer Cores 2 Disks legacy program (Evans et al. 2003) and 20 of our 24 targets come from that work. A Spitzer color (where [x] indicates the magnitude in the $x \mu \mathrm{m}$ band) of [3.6]$[4.5]<0.25$ indicates the flux deficit characteristic of the nearinfrared/inner opacity holes of a TD, while a color of [3.6][24] $>1.5$ ensures that all of the targets have significant midIR excesses at $24 \mu \mathrm{m}$. We included an additional four Oph targets and seven from other regions, all of which were identified by other authors using the same criteria as C10 (Andrews \& Williams 2007a; Geers et al. 2007; Furlan et al. 2009).

The Oph targets in this study are shown in Figure 1 in relation to other surveys of YSOs in the region. Table 1 lists each target in our sample, its stellar association, coordinates, SpT, and $V, R$, and $J$ magnitudes. Targets that have known wide stellar companions are also noted. The SpT distribution of our sample is shown in Figure 2 and is dominated by late-K to mid-M stars with a median SpT of M2.

\subsection{Observations}

We acquired high-resolution optical spectra using the Magellan Inamori Kyocera Echelle (MIKE; Bernstein et al. 2003) spectrograph at the Magellan (Clay) $6.5 \mathrm{~m}$ telescope. Twenty-one of our targets were observed twice, two or three days apart during 2010 June 18-23 (UT100618$\mathrm{UT} 100623)^{7}$ and then nearly a year later on 2011 June $14-15$ (UT110614-UT110615). Two targets were observed a third time on 2012 May 9-10 (UT120509-UT120510) and another on 2012 January 17 (UT120117). The complete $\log$ of our observations is recorded in Table 2. We used the red arm of the spectrograph and the $0 . " 5$ slit to obtain a spectrum from 4900 to $9150 \AA$ with a spectral resolution of $\sim 47,000$. Total exposure times ranged from 2 to 30 minutes per target depending on stellar brightness to achieve a typical signal-to-noise ratio

Throughout this paper we use a date format of UTYYMMDD. 
Table 1

Transition Disk Targets

\begin{tabular}{|c|c|c|c|c|c|c|c|c|c|c|}
\hline Name & $\begin{array}{l}\text { Other } \\
\text { Identifier }\end{array}$ & Association & $\begin{array}{c}\alpha(\mathrm{J} 2000) \\
\mathrm{h} \mathrm{m} \mathrm{s}\end{array}$ & $\begin{array}{l}\delta(\mathrm{J} 2000) \\
0,11\end{array}$ & $\overline{\mathrm{SpT}}$ & $\begin{array}{c}V \\
\mathrm{mag}\end{array}$ & $\begin{array}{c}R \\
\mathrm{mag}\end{array}$ & $\begin{array}{c}\text { (2MASS) } \\
\text { mag }\end{array}$ & $\begin{array}{c}\text { Known Visual Multiplicity } \\
\text { (Separation in ") }\end{array}$ & Source $^{\mathrm{b}}$ \\
\hline SZ Cha & & Cha & 105816.8 & -771717.1 & K0 & 12.68 & $\cdots$ & 9.25 & Binary $(\sim 5)$ & $(1),(2),(3),(4)$ \\
\hline $\mathrm{T} 25$ & Sz 18 & Cha & 110719.1 & -760304.8 & M2.5 & 15.35 & 13.7 & 10.96 & $\ldots$ & $(1),(5)$ \\
\hline T35 & Sz 27 & Cha & 110839.0 & -771604.2 & K8 & $\ldots$ & 15.82 & 11.17 & $\ldots$ & $(1),(5)$ \\
\hline RX J1852.3-3700 & & $\mathrm{CrA}$ & 185217.3 & -370012.0 & K7 & 12.19 & $\ldots$ & 9.77 & $\ldots$ & (7), (8) \\
\hline CrA-4111 & & CrA & 190120.8 & -370303.0 & M4.5 & $\ldots$ & $\ldots$ & 13.23 & $\ldots$ & (9) \\
\hline G-49 & CrA 468 & Cor & 190149.4 & -370028.0 & M4 & $\ldots$ & 16.6 & 12.5 & $\ldots$ & (9), (10) \\
\hline G-102 & CrA 133 & Cor & 190125.6 & -370453.0 & M5 & $\cdots$ & 15.3 & 12.36 & $\cdots$ & (9), (5) \\
\hline SSTc2d J162118.5-225458 & $\ldots$ & Oph & 162118.5 & -225458.0 & M2 & $\cdots$ & $\ldots$ & 11.45 & $\cdots$ & (11) \\
\hline SSTc2d J162218.5-232148 & $\ldots$ & Oph & 162218.5 & -232148.0 & K5 & 12.67 & $\ldots$ & 9.52 & $\ldots$ & $(11),(12)$ \\
\hline SSTc2d J162245.4-243124 & $\ldots$ & Oph & 162245.4 & -243124.0 & M3 & $\cdots$ & 14.2 & 10.38 & $\cdots$ & $(11),(5)$ \\
\hline SSTc2d J162309.2-241705 & $\ldots$ & Oph & 162309.3 & -241703.0 & M? & 12.75 & 14.2 & 10.32 & $\ldots$ & $(1),(5)$ \\
\hline SSTc2d J162332.8-225847 & $\ldots$ & Oph & 162332.8 & -225847.0 & M5 & & 15.7 & 11.49 & $\ldots$ & $(11),(5)$ \\
\hline SSTc2d J162336.1-240221 & $\cdots$ & Oph & 162336.1 & -24221.0 & M5 & 14.68 & $\cdots$ & 11.53 & $\cdots$ & $(11),(13)$ \\
\hline SSTc2d J162506.9-235050 & $\cdots$ & Oph & 162506.9 & -235050.0 & M3 & $\cdots$ & $\ldots$ & 11.05 & & (11) \\
\hline SSTc2d J162623.7-244314 & DoAr 25 & Oph & 162623.7 & -244314.0 & K5 & $\cdots$ & 12.65 & 9.39 & $\cdots$ & $(11),(14)$ \\
\hline SSTc2d J162646.4-241160 & $\cdots$ & Oph & 162646.4 & -241160.0 & G5 & $\cdots$ & 13.9 & 9.68 & Binary $(0.58)$ & (11), (14), (15) \\
\hline DoAr28 & Haro $1-8$ & Oph & 162647.4 & -231452.2 & $\mathrm{~K}$ ? & 13.84 & 12.1 & 9.89 & $\cdots$ & $(16),(17),(5)$ \\
\hline SR21A & $\cdots$ & Oph & 162710.3 & -241912.7 & G3 & 14.1 & $\cdots$ & 8.75 & Binary (896) & (18), (19), (20) \\
\hline SSTc2d J162738.3-235732 & DoAr 32 & Oph & 162738.3 & -235732.0 & K5 & $\cdots$ & 13.66 & 9.91 & Triple $(?)^{\mathrm{c}}$ & $(11),(14),(15),(21)$ \\
\hline SSTc2d J162739.0-235818 & DoAr 33 & Oph & 162739.0 & -235818.0 & K6 & & 13.24 & 9.9 & Triple $(?)^{\mathrm{c}}$ & $(11),(14),(15),(21)$ \\
\hline SSTc2d J162740.3-242204 & DoAr 34 & Oph & 162740.3 & -242204.0 & K5 & 11.5 & 11.1 & 8.44 & $\cdots$ & $(11),(22)$ \\
\hline SSTc2d J162802.6-235504 & $\cdots$ & Oph & 162802.6 & -235504.0 & M3 & $\cdots$ & & 11.76 & $\cdots$ & (11) \\
\hline SSTc2d J162821.5-242155 & $\cdots$ & Oph & 162821.5 & -242155.0 & M3 & $\cdots$ & 16.97 & 12.08 & $\cdots$ & $(11),(14)$ \\
\hline SSTc2d J162854.1-244744 & $\cdots$ & Oph & 162854.1 & -244744.0 & M2 & $\cdots$ & 15.24 & 10.68 & $\cdots$ & $(11),(14)$ \\
\hline SSTc2d J163020.0-233108 & $\ldots$ & Oph & 163020.0 & -233108.0 & M4 & $\ldots$ & $\cdots$ & 11.32 & $\cdots$ & (11) \\
\hline SSTc2d J163033.9-242806 & $\ldots$ & Oph & 163033.9 & -242806.0 & M4 & $\ldots$ & $\ldots$ & 11.63 & $\ldots$ & (11) \\
\hline SSTc2d J163145.4-244307 & $\ldots$ & Oph & 163145.4 & -244307.0 & M4 & $\ldots$ & $\ldots$ & 11.8 & $\ldots$ & (11) \\
\hline SSTc2d J163154.4-250349 & $\cdots$ & Oph & 163154.4 & -250349.0 & M4 & $\cdots$ & $\cdots$ & 11.78 & $\cdots$ & (11) \\
\hline SSTc2d J163154.7-250324 & $\mathrm{H} \alpha 74$ & Oph & 163154.7 & -250324.0 & K7 & 12.64 & 13.3 & 10.14 & $\cdots$ & $(11),(5)$ \\
\hline SSTc2d J163205.5-250236 & $\ldots$ & Oph & 163205.5 & -250236.0 & M2 & $\ldots$ & 15.5 & 11.65 & $\ldots$ & $(11),(5)$ \\
\hline SSTc2d J163355.6-244205 & $\ldots$ & Oph & 163355.6 & -244205.0 & K7 & $\ldots$ & 14.1 & 10.46 & $\ldots$ & $(11),(5)$ \\
\hline
\end{tabular}

Notes.

${ }^{\text {a }}$ Binary companions all lie outside of the TD.

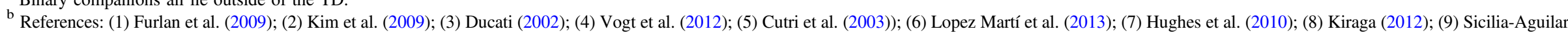

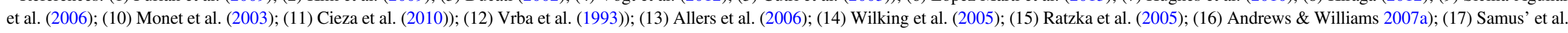

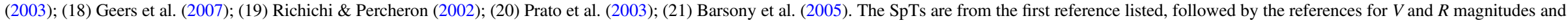
then visual multiplicity, if applicable. All $J$ magnitudes are from the 2MASS catalog (Skrutskie et al. 2006).

${ }^{\mathrm{c}}$ SSTc2d J162738.3-235732 and SSTc2d J162739.0-235818 are both possible members of the same triple system about primary ROXs 30 A $\left(16^{\mathrm{h}} 27^{\mathrm{m}} 37^{\mathrm{s}} .0-23^{\circ} 59^{\prime} 32^{\prime \prime}\right.$; Ratzka et al. 2005). 


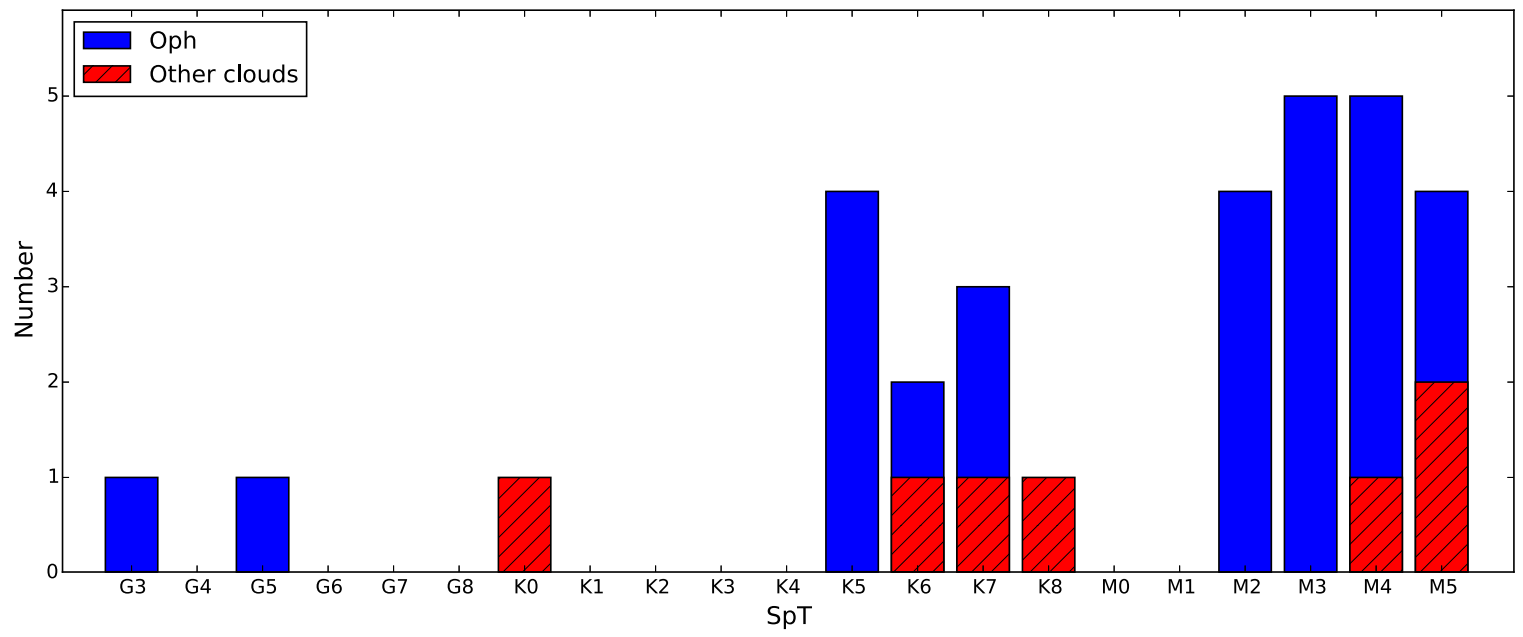

Figure 2. Spectral type distribution of the TD sample. All of the targets have ages $\leqslant 10 \mathrm{Myr}$.

$(\mathrm{S} / \mathrm{N})$ of $\approx 50$ per resolution element at $8000 \AA$. For the faintest stars, the $\mathrm{S} / \mathrm{N}$ was roughly $10-20$.

Data were reduced using the facility pipeline (Kelson 2003). Each stellar exposure was bias-subtracted and flat-fielded for pixel-to-pixel sensitivity variations. After optimal extraction, spectra were wavelength calibrated with a ThAr arc taken within an hour of the stellar exposure. To correct for instrumental drift, the telluric molecular oxygen $\mathrm{A}$ band (7619-7657 $\AA$ ) was used to align the MIKE spectra to an average precision of $70 \mathrm{~m} \mathrm{~s}^{-1}$. We then corrected for the heliocentric velocity.

\section{RESULTS}

\subsection{Radial Velocities}

Each target spectrum was cross-correlated against an RV standard star best matched to its SpT and observed on the same night. We used the IRAF $^{8}$ fxcor routine (Wyatt 1985; Fitzpatrick 1993) to obtain RVs and search for single- and double-lined spectroscopic binaries (SB1s and SB2s, respectively). The standards and their published RVs are listed in Table 3. We used orders 39, 40, 43, 44, 46, 48, 49, 51, and 53, which ranged from 6400 to $8900 \AA$, for the cross-correlation to avoid telluric absorption lines and very low $\mathrm{S} / \mathrm{N}$ regions. The $\mathrm{RVs}$ reported in Table 2 are the averages of those measured for each order weighted by the average $\mathrm{S} / \mathrm{N}$ in each order. Table 2 also lists the standard star used, and Li I and $\mathrm{H} \alpha$ EWs (see Appendix A.1 for details of $\mathrm{Li}$ I and $\mathrm{H} \alpha$ analysis).

The uncertainties in our RV measurements include the standard error on the mean RV across the cross-correlated apertures (typically $\sim 400 \mathrm{~ms}^{-1}$ ), an uncertainty of $300 \mathrm{~ms}^{-1}$ to account for RV distortions due to star spots on young stars (Hatzes 2002; Mohanty et al. 2002; Berdyugina 2005; Desort et al. 2007), and an additional $400 \mathrm{~ms}^{-1}$ to account for the zeropoint uncertainty in the RVs of our standard stars (see Table 3), all added in quadrature. In all cases, the systematic uncertainties from possible spot activity and the zero-point dominate the final uncertainties. Note that $\Delta(\mathrm{RV})$, the difference in RVs

\footnotetext{
8 IRAF is distributed by the National Optical Astronomy Observatories, which are operated by the Association of Universities for Research in Astronomy, Inc., under a cooperative agreement with the National Science Foundation.
}

between epochs, need not include the uncertainty in the RV of the standards as the same standards were used.

The largest systematic uncertainties in these results will come from mismatched SpTs between the TD objects and the RV standard stars. While we endeavoured to match SpTs as closely as possible, the lack of $\mathrm{G}$ or $\mathrm{K}$ stars among our RV standards implies that for $\mathrm{G}$ and K-type TDs, systematics may remain unaccounted for. However, this should only affect SZ Cha (K0), SSTc2d SSTc2d J162646.4-241160 (G5) and SR21A (G3-K?; a discussion of its SpT can be found in Appendix A.2), since late K-type stars can be well matched by early M-type standards. Indeed, we see that the uncertainties on the RVs of SZ Cha are larger $(\sim 2 \times)$ than the average uncertainties.

The average RV of our Oph targets is $-6.6 \mathrm{~km} \mathrm{~s}^{-1}$ with a velocity dispersion of $1.3 \mathrm{~km} \mathrm{~s}^{-1}$, which agrees well with other measurements for the RV of the cloud: $-5.64 \pm 2.57 \mathrm{~km} \mathrm{~s}^{-1}$ from Kurosawa et al. (2006) and $-6.27 \pm 1.48 \mathrm{~km} \mathrm{~s}^{-1}$ from Prato (2007). There are no overlapping targets between our sample and those studies.

Our average $\mathrm{RV}$ for Cha targets is $15 \pm 1 \mathrm{~km} \mathrm{~s}^{-1}$, which is in good agreement with James et al. (2006), who report an average of $12.8 \pm 3.6 \mathrm{~km} \mathrm{~s}^{-1}$. However, our measurements of the average $\mathrm{RV}$ of $\mathrm{CrA}+\mathrm{Cor},-3 \pm 1 \mathrm{~km} \mathrm{~s}^{-1}$ differs by $\sim 1.5 \sigma$ from James et al.'s findings of $-1.1 \pm 0.5 \mathrm{~km} \mathrm{~s}^{-1}$. It is, however, in good agreement with Neuhäuser et al. (2000), who find an average RV of $-2.6 \pm 1.4 \mathrm{~km} \mathrm{~s}^{-1}$ for ROSAT-selected $\mathrm{T}$ Tauri stars in CrA.

The close agreement in mean RV values within our sample implies that any single RV measurement that deviates by more than $3 \sigma$ from the region average would be a highly probable RV-variable SB1 (Kurosawa et al. 2006; Prato 2007). We find no such targets in our sample.

\subsection{Rotational Velocities}

We determined rotational velocities $(v \sin i)$ following the "Fourier Method" (e.g., Carroll 1933; Gray 1976, 2005; Reiners et al. 2001; Simón-Díaz \& Herrero 2007). Using telluric lines, we determined a small amount of instrumental broadening $(\sim 0.2 \AA)$, which we absorbed into model lines of zero rotation. We extracted the excess broadening due to rotation using the relatively high S/N Li I doublet at $6708 \AA$ and Ca I at $6718 \AA$. While forbidden atomic transitions such as [Fe III] do not run the 
Table 2

Observation Log and Measured Properties of TD Objects

\begin{tabular}{|c|c|c|c|c|c|c|}
\hline $\begin{array}{l}\text { Target } \\
\end{array}$ & $\begin{array}{l}\text { Date observed } \\
\text { yyyy } / \mathrm{mm} / \mathrm{dd}\end{array}$ & $\begin{array}{l}\text { Standard } \\
\text { Star Used }\end{array}$ & $\begin{array}{c}\mathrm{RV} \\
\mathrm{km} \mathrm{s}^{-1}\end{array}$ & $\begin{array}{c}\mathrm{Li} \text { I } \mathrm{EW}^{\mathrm{a}} \\
\AA\end{array}$ & $\begin{array}{c}\mathrm{H} \alpha \mathrm{EW}^{\mathrm{a}} \\
\AA\end{array}$ & $\begin{array}{c}\mathrm{H} \alpha 10 \%{ }^{\mathrm{b}} \\
\mathrm{km} \mathrm{s}^{-1}\end{array}$ \\
\hline \multirow[t]{4}{*}{$\overline{\mathrm{SZ} \mathrm{Cha}}{ }^{\mathrm{c}}$} & 2010 Jun 18 & GJ 908 & $12.1 \pm 0.5$ & $0.36 \pm 0.04$ & $-3.68 \pm 0.03$ & $270 \pm 25$ \\
\hline & 2010 Jun 21 & GJ 908 & $14.0 \pm 1.5$ & $0.38 \pm 0.02$ & $-26.6 \pm 0.5$ & $479 \pm 47$ \\
\hline & 2011 Jun 14 & GJ 514 & $17.0 \pm 1.3$ & $0.42 \pm 0.05$ & $-15.7 \pm 0.9$ & $478 \pm 43$ \\
\hline & mean & & $14.0 \pm 2.0$ & $0.39 \pm 0.07$ & $-14 \pm 2$ & $409 \pm 69$ \\
\hline \multirow[t]{4}{*}{$\mathrm{T} 25^{\mathrm{c}}$} & 2010 Jun 18 & GJ 908 & $15.3 \pm 0.3$ & $0.55 \pm 0.02$ & $-10.7 \pm 0.8$ & $327 \pm 29$ \\
\hline & 2010 Jun 21 & GJ 908 & $15.6 \pm 0.3$ & $0.58 \pm 0.02$ & $-11 \pm 2$ & $347 \pm 34$ \\
\hline & 2011 Jun 14 & GJ 514 & $16.0 \pm 0.3$ & $0.60 \pm 0.04$ & $-21 \pm 1$ & $444 \pm 49$ \\
\hline & mean & & $15.7 \pm 0.6$ & $0.58 \pm 0.05$ & $-15 \pm 1$ & $373 \pm 66$ \\
\hline \multirow[t]{3}{*}{$\mathrm{T} 35$} & 2010 Jun 21 & GJ 908 & $16.2 \pm 0.6$ & $0.49 \pm 0.03$ & $-118 \pm 15$ & $482 \pm 45$ \\
\hline & 2011 Jun 14 & GJ 699 & $16.8 \pm 0.6$ & $0.41 \pm 0.08$ & $-128 \pm 16$ & $520 \pm 55$ \\
\hline & mean & & $16.5 \pm 0.8$ & $0.45 \pm 0.09$ & $-123 \pm 22$ & $501 \pm 71$ \\
\hline \multirow[t]{4}{*}{ RX J1852.3-3700 d } & 2010 Jun 19 & GJ 908 & $-1.5 \pm 0.3$ & $0.5 \pm 0.1$ & $-45 \pm 6$ & $320 \pm 30$ \\
\hline & 2010 Jun 22 & GJ 699 & $-2.6 \pm 0.5$ & $0.5 \pm 0.1$ & $-33 \pm 2$ & $325 \pm 33$ \\
\hline & 2011 Jun 14 & GJ 514 & $-3.0 \pm 0.3$ & $0.48 \pm 0.01$ & $-27 \pm 3$ & $326 \pm 35$ \\
\hline & mean & & $-2.4 \pm 0.6$ & $0.5 \pm 0.2$ & $-35 \pm 7$ & $324 \pm 57$ \\
\hline $\mathrm{CrA}-4111$ & 2010 Jun 23 & GJ 699 & $-5.2 \pm 0.3$ & $<0.16$ & $-16 \pm 4$ & $135 \pm 14$ \\
\hline \multirow{4}{*}{ G-49 } & 2011 Jun 15 & GJ 699 & $-2.7 \pm 0.3$ & $0.51 \pm 0.04$ & $-3 \pm 2$ & $92 \pm 9$ \\
\hline & 2012 May 09 & GJ 699 & $-2.6 \pm 0.3$ & $0.62 \pm 0.01$ & $-4.32 \pm 0.05$ & $106 \pm 10$ \\
\hline & 2012 May 10 & GJ 699 & $-2.8 \pm 0.3$ & $0.62 \pm 0.01$ & $-4.71 \pm 0.02$ & $101 \pm 9$ \\
\hline & mean & & $-2.7 \pm 0.6$ & $0.55 \pm 0.09$ & $-4 \pm 2$ & $100 \pm 16$ \\
\hline \multirow[t]{4}{*}{ G-102 } & 2011 Jun 15 & GJ 699 & $-2.8 \pm 0.5$ & $0.61 \pm 0.03$ & $-14 \pm 4$ & $189 \pm 18$ \\
\hline & 2012 May 09 & GJ 699 & $-2.9 \pm 0.3$ & $0.66 \pm 0.01$ & $-20 \pm 2$ & $153 \pm 14$ \\
\hline & 2012 May 10 & GJ 699 & $-1.0 \pm 0.3$ & $0.67 \pm 0.01$ & $-17 \pm 1$ & $152 \pm 7$ \\
\hline & mean & & $-2.2 \pm 0.7$ & $0.65 \pm 0.03$ & $-17 \pm 4$ & $165 \pm 24$ \\
\hline \multirow[t]{3}{*}{ SSTc2d J162118.5-225458 } & 2010 Jun 21 & GJ 908 & $-6.3 \pm 0.4$ & $0.48 \pm 0.01$ & $-14.3 \pm 0.4$ & $336 \pm 36$ \\
\hline & 2010 Jun 22 & GJ 908 & $-6.6 \pm 0.4$ & $0.5 \pm 0.01$ & $-8.9 \pm 0.7$ & $285 \pm 30$ \\
\hline & mean & & $-6.4 \pm 0.5$ & $0.49 \pm 0.01$ & $-11.6 \pm 0.8$ & $310 \pm 47$ \\
\hline \multirow[t]{4}{*}{ SSTc2d J162218.5-232148 } & 2010 Jun 19 & GJ 908 & $-7.9 \pm 0.4$ & $0.4 \pm 0.2$ & $-8.2 \pm 0.2$ & $362 \pm 40$ \\
\hline & 2010 Jun 21 & GJ 908 & $-9.7 \pm 0.4$ & $0.4 \pm 0.1$ & $-15 \pm 1$ & $551 \pm 53$ \\
\hline & 2011 Jun 14 & GJ 514 & $-7.5 \pm 0.4$ & $0.42 \pm 0.03$ & $-12.5 \pm 0.7$ & $378 \pm 37$ \\
\hline & mean & & $-8.4 \pm 0.7$ & $0.4 \pm 0.2$ & $-12 \pm 2$ & $430 \pm 76$ \\
\hline \multirow[t]{4}{*}{ SSTc2d J162245.4-243124 } & 2010 Jun 20 & GJ 699 & $-5.3 \pm 0.3$ & $0.5 \pm 0.4$ & $-4.6 \pm 0.4$ & $120 \pm 13$ \\
\hline & 2010 Jun 22 & GJ 699 & $-5.4 \pm 0.3$ & $0.51 \pm 0.01$ & $-5.1 \pm 0.8$ & $124 \pm 12$ \\
\hline & 2011 Jun 14 & GJ 699 & $-4.0 \pm 0.3$ & $0.55 \pm 0.05$ & $-4.2 \pm 0.8$ & $115 \pm 4$ \\
\hline & mean & & $-4.8 \pm 0.6$ & $0.5 \pm 0.4$ & $-5 \pm 1$ & $120 \pm 18$ \\
\hline \multirow[t]{4}{*}{ SSTc2d J162309.2-241705 } & 2010 Jun 20 & GJ 908 & $-3.8 \pm 0.4$ & $0.28 \pm 0.06$ & $-17.3 \pm 0.5$ & $419 \pm 46$ \\
\hline & 2010 Jun 22 & GJ 908 & $-2.9 \pm 0.4$ & $0.29 \pm 0.01$ & $-16.3 \pm 0.8$ & $365 \pm 30$ \\
\hline & 2011 Jun 14 & GJ 699 & $-3.6 \pm 0.4$ & $0.29 \pm 0.01$ & $-14 \pm 1$ & $363 \pm 35$ \\
\hline & mean & & $-3.4 \pm 0.7$ & $0.29 \pm 0.06$ & $-16 \pm 2$ & $382 \pm 65$ \\
\hline \multirow[t]{3}{*}{ SSTc2d J162332.8-225847 } & 2010 Jun 20 & GJ 699 & $-6.9 \pm 0.3$ & $0.54 \pm 0.01$ & $-13 \pm 3$ & $153 \pm 15$ \\
\hline & 2010 Jun 22 & GJ 699 & $-7.0 \pm 0.3$ & $0.44 \pm 0.03$ & $-11 \pm 3$ & $122 \pm 13$ \\
\hline & mean & & $-6.9 \pm 0.4$ & $0.49 \pm 0.04$ & $-12 \pm 4$ & $137 \pm 20$ \\
\hline \multirow[t]{3}{*}{ SSTc2d J162336.1-240221 } & 2010 Jun 20 & GJ 699 & $-6.7 \pm 0.3$ & $<0.28$ & $-12 \pm 3$ & $93 \pm 8$ \\
\hline & 2010 Jun 22 & GJ 699 & $-7.3 \pm 0.3$ & $0.36 \pm 0.01$ & $-8 \pm 1$ & $113 \pm 11$ \\
\hline & mean & & $-7.0 \pm 0.4$ & $0.4 \pm 0.3$ & $-10 \pm 3$ & $103 \pm 14$ \\
\hline \multirow[t]{4}{*}{ SSTc2d J162506.9-235050 } & 2010 Jun 20 & GJ 699 & $-6.5 \pm 0.3$ & $0.51 \pm 0.01$ & $-9 \pm 1$ & $259 \pm 26$ \\
\hline & 2010 Jun 22 & GJ 699 & $-6.9 \pm 0.3$ & $0.53 \pm 0.01$ & $-12 \pm 3$ & $341 \pm 34$ \\
\hline & 2011 Jun 14 & GJ 699 & $-7.0 \pm 0.3$ & $0.57 \pm 0.01$ & $-14 \pm 4$ & $383 \pm 48$ \\
\hline & mean & & $-6.8 \pm 0.6$ & $0.53 \pm 0.01$ & $-12 \pm 5$ & $328 \pm 64$ \\
\hline \multirow[t]{3}{*}{ SSTc2d J162623.7-244314 } & 2012 Jun 21 & GJ 908 & $-7.2 \pm 0.4$ & $0.5 \pm 0.2$ & $-4.5 \pm 0.4$ & $239 \pm 25$ \\
\hline & 2011 Jun 14 & GJ 908 & $-6.7 \pm 0.4$ & $0.56 \pm 0.02$ & $-11 \pm 1$ & $467 \pm 47$ \\
\hline & mean & & $-6.9 \pm 0.6$ & $0.5 \pm 0.2$ & $-8 \pm 1$ & $353 \pm 53$ \\
\hline SSTc2d J162646.4-241160 & 2010 Jun 20 & GJ 908 & $-6.3 \pm 0.4$ & $0.5 \pm 0.1$ & $-11.7 \pm 0.4$ & $346 \pm 31$ \\
\hline & 2010 Jun 22 & GJ 908 & $-6.6 \pm 0.3$ & $0.4 \pm 0.2$ & $-14.7 \pm 0.8$ & $368 \pm 36$ \\
\hline & 2011 Jun 14 & GJ 514 & $-6.2 \pm 0.4$ & $0.4 \pm 0.1$ & $-17 \pm 2$ & $356 \pm 34$ \\
\hline & mean & & $-6.4 \pm 0.7$ & $0.4 \pm 0.3$ & $-14.38 \pm 2.2$ & $357 \pm 58$ \\
\hline DoAr28 & 2010 Jun 20 & GJ 908 & $-8.6 \pm 0.3$ & $0.45 \pm 0.01$ & $-25 \pm 2$ & $451 \pm 40$ \\
\hline & 2010 Jun 22 & GJ 908 & $-8.0 \pm 0.4$ & $0.4 \pm 0.2$ & $-29 \pm 2$ & $441 \pm 47$ \\
\hline & 2011 Jun 14 & GJ 514 & $-7.9 \pm 0.3$ & $0.4 \pm 0.2$ & $-28 \pm 1$ & $436 \pm 40$ \\
\hline & mean & & $-8.2 \pm 0.6$ & $0.4 \pm 0.3$ & $-27 \pm 3$ & $443 \pm 74$ \\
\hline SR21A & 2011 Jun 14 & GJ 908 & $-3.6 \pm 0.5$ & $0.13 \pm 0.02$ & $1.1 \pm 0.15^{\mathrm{e}}$ & $\ldots$ \\
\hline SSTc2d J162738.3-235732 & 2010 Jun 19 & GJ 908 & $-7.4 \pm 0.3$ & $0.47 \pm 0.01$ & $-16 \pm 2$ & $291 \pm 33$ \\
\hline & 2010 Jun 22 & GJ 908 & $-6.5 \pm 0.3$ & $0.49 \pm 0.02$ & $-14 \pm 3$ & $275 \pm 29$ \\
\hline
\end{tabular}


Table 2

(Continued)

\begin{tabular}{|c|c|c|c|c|c|c|}
\hline Target & $\begin{array}{l}\text { Date observed } \\
\text { yyyy } / \mathrm{mm} / \mathrm{dd}\end{array}$ & $\begin{array}{c}\text { Standard } \\
\text { Star Used } \\
\end{array}$ & $\begin{array}{c}\mathrm{RV} \\
\mathrm{km} \mathrm{s}^{-1}\end{array}$ & $\begin{array}{c}\mathrm{Li} \text { I } \mathrm{EW}^{\mathrm{a}} \\
\AA \\
\AA\end{array}$ & $\begin{array}{c}\mathrm{H} \alpha \mathrm{EW}^{\mathrm{a}} \\
\AA\end{array}$ & $\begin{array}{c}\mathrm{H} \alpha 10 \%^{\mathrm{b}} \\
\mathrm{km} \mathrm{s}^{-1} \\
\end{array}$ \\
\hline & 2011 Jun 14 & GJ 514 & $-6.4 \pm 0.3$ & $0.5 \pm 0.05$ & $-13.4 \pm 0.9$ & $292 \pm 29$ \\
\hline & mean & & $-6.8 \pm 0.6$ & $0.49 \pm 0.05$ & $-15 \pm 4$ & $286 \pm 53$ \\
\hline \multirow{4}{*}{ SSTc2d J162739.0-235818 } & 2010 Jun 19 & GJ 699 & $-7.7 \pm 0.5$ & $0.49 \pm 0.01$ & $-22 \pm 1$ & $373 \pm 37$ \\
\hline & 2010 Jun 21 & GJ 699 & $-7.3 \pm 0.7$ & $0.52 \pm 0.01$ & $-13 \pm 4$ & $282 \pm 28$ \\
\hline & 2011 Jun 14 & GJ 514 & $-7.0 \pm 0.4$ & $0.54 \pm 0.01$ & $-9.9 \pm 0.2$ & $261 \pm 27$ \\
\hline & mean & & $-7.3 \pm 0.9$ & $0.51 \pm 0.02$ & $-15 \pm 4$ & $306 \pm 54$ \\
\hline \multirow[t]{3}{*}{ SSTc2d J162740.3-242204 } & 2010 Jun 22 & GJ 908 & $-6.0 \pm 0.4$ & $0.5 \pm 0.1$ & $-10.3 \pm 0.2$ & $289 \pm 29$ \\
\hline & 2011 Jun 14 & GJ 514 & $-7.2 \pm 0.3$ & $0.51 \pm 0.01$ & $-10.77 \pm 0.09$ & $362 \pm 32$ \\
\hline & mean & & $-6.6 \pm 0.5$ & $0.5 \pm 0.1$ & $-10.5 \pm 0.2$ & $325 \pm 43$ \\
\hline \multirow[t]{3}{*}{ SSTc2d J162802.6-235504 } & 2010 Jun 20 & GJ 908 & $-7.3 \pm 0.4$ & $0.51 \pm 0.04$ & $<1$ & $\cdots$ \\
\hline & 2010 Jun 22 & GJ 908 & $-6.6 \pm 0.3$ & $0.57 \pm 0.08$ & $-3.7 \pm 0.2$ & $77 \pm 6$ \\
\hline & mean & & $-6.9 \pm 0.5$ & $0.54 \pm 0.09$ & $-3.5 \pm 0.9$ & $77 \pm 6$ \\
\hline \multirow[t]{3}{*}{ SSTc2d J162821.5-242155 } & 2010 Jun 21 & GJ 699 & $-7.1 \pm 0.3$ & $0.4 \pm 0.2$ & $-2.9 \pm 0.6$ & $82 \pm 7$ \\
\hline & 2010 Jun 22 & GJ 699 & $-7.5 \pm 0.3$ & $0.4 \pm 0.2$ & $-3 \pm 1$ & $95 \pm 5$ \\
\hline & mean & & $-7.3 \pm 0.5$ & $0.4 \pm 0.3$ & $-3 \pm 1$ & $88 \pm 9$ \\
\hline \multirow[t]{4}{*}{ SSTc2d J162854.1-244744 } & 2010 Jun 20 & GJ 908 & $-5.0 \pm 0.3$ & $0.58 \pm 0.02$ & $-21.7 \pm 0.4$ & $401 \pm 37$ \\
\hline & 2010 Jun 22 & GJ 908 & $-5.1 \pm 0.3$ & $0.59 \pm 0.02$ & $-24 \pm 6$ & $314 \pm 29$ \\
\hline & 2011 Jun 14 & GJ 514 & $-4.4 \pm 0.3$ & $0.6 \pm 0.3$ & $-65 \pm 13$ & $423 \pm 52$ \\
\hline & mean & & $-4.8 \pm 0.6$ & $0.6 \pm 0.3$ & $-37 \pm 14$ & $379 \pm 70$ \\
\hline \multirow[t]{3}{*}{ SSTc2d J163020.0-233108 } & 2010 Jun 21 & GJ 908 & $-7.2 \pm 0.4$ & $0.57 \pm 0.75$ & $-2.1 \pm 0.4$ & $87 \pm 7$ \\
\hline & 2010 Jun 22 & GJ 699 & $-6.7 \pm 0.3$ & $0.56 \pm 0.57$ & $-1.6 \pm 0.4$ & $83 \pm 7$ \\
\hline & mean & & $-7.0 \pm 0.5$ & $0.57 \pm 0.94$ & $-1.8 \pm 0.6$ & $85 \pm 10$ \\
\hline \multirow[t]{3}{*}{ SSTc2d J163033.9-242806 } & 2010 Jun 20 & GJ 699 & $-6.5 \pm 0.5$ & $1.3 \pm 0.2$ & $-19 \pm 3$ & $332 \pm 33$ \\
\hline & 2010 Jun 22 & GJ 699 & $-6.1 \pm 0.4$ & $0.51 \pm 0.08$ & $-17 \pm 1$ & $247 \pm 24$ \\
\hline & mean & & $-6.3 \pm 0.6$ & $0.9 \pm 0.2$ & $-18 \pm 4$ & $290 \pm 41$ \\
\hline \multirow[t]{4}{*}{ SSTc2d J163145.4-244307 } & 2010 Jun 21 & GJ 699 & $-5.0 \pm 0.4$ & $0.5 \pm 0.4$ & $-12.2 \pm 0.2$ & $455 \pm 49$ \\
\hline & 2010 Jun 22 & GJ 699 & $-2.4 \pm 0.4$ & $0.3 \pm 0.2$ & $-37 \pm 2$ & $446 \pm 39$ \\
\hline & 2011 Jun 14 & GJ 699 & $-4.8 \pm 0.3$ & $0.5 \pm 0.05$ & $-60 \pm 9$ & $364 \pm 37$ \\
\hline & mean & & $-4.1 \pm 0.7$ & $0.4 \pm 0.4$ & $-36 \pm 9$ & $422 \pm 73$ \\
\hline \multirow[t]{3}{*}{ SSTc2d J163154.4-250349 } & 2010 Jun 21 & GJ 699 & $-4.6 \pm 0.4$ & $0.3 \pm 0.1$ & $-106 \pm 29$ & $374 \pm 43$ \\
\hline & 2011 Jun 14 & GJ 514 & $-3.7 \pm 0.3$ & $0.34 \pm 0.07$ & $-139 \pm 33$ & $402 \pm 35$ \\
\hline & mean & & $-4.1 \pm 0.5$ & $0.3 \pm 0.1$ & $-123 \pm 44$ & $388 \pm 56$ \\
\hline \multirow[t]{4}{*}{ SSTc2d J163154.7-250324 (SB2) } & 2010 Jun 20 & GJ 699 & $-12.1 \pm 1.0$ & $0.56 \pm 0.01$ & $-8.4 \pm 0.2$ & $394 \pm 38$ \\
\hline & 2010 Jun 22 & GJ 699 & $-16.4 \pm 1.2$ & $0.5 \pm 0.2$ & $-6 \pm 1$ & $301 \pm 27$ \\
\hline & 2011 Jun 14 & GJ 514 & $-5.4 \pm 0.5$ & $0.5 \pm 0.2$ & $-7 \pm 2$ & $397 \pm 39$ \\
\hline & mean & & $-11 \pm 2$ & $0.5 \pm 0.2$ & $-7 \pm 2$ & $364 \pm 61$ \\
\hline \multirow[t]{3}{*}{ SSTc2d J163205.5-250236 } & 2010 Jun 21 & GJ 908 & $-6.7 \pm 0.3$ & $0.50 \pm 0.09$ & $-34 \pm 2$ & $461 \pm 43$ \\
\hline & 2012 Jan 17 & GJ 908 & $-6.0 \pm 0.4$ & $0.51 \pm 0.01$ & $-25 \pm 2$ & $427 \pm 39$ \\
\hline & mean & & $-6.4 \pm 0.5$ & $0.51 \pm 0.09$ & $-29 \pm 3$ & $444 \pm 58$ \\
\hline \multirow[t]{3}{*}{ SSTc2d J163355.6-244205 } & 2010 Jun 22 & GJ 699 & $-6.1 \pm 0.4$ & $0.52 \pm 0.02$ & $-2.7 \pm 0.6$ & $255 \pm 23$ \\
\hline & 2011 Jun 14 & GJ 514 & $-5.8 \pm 0.3$ & $0.53 \pm 0.02$ & $-7 \pm 3$ & $283 \pm 31$ \\
\hline & mean & & $-6.0 \pm 0.5$ & $0.53 \pm 0.03$ & $-5 \pm 3$ & $269 \pm 39$ \\
\hline
\end{tabular}

Notes.

a If the spectral line could not be distinguished from the continuum, a $2 \sigma$ limit is shown.

${ }^{\mathrm{b}} \mathrm{H} \alpha 10 \%$ velocity width (White \& Basri 2003) variability is due to stellar activity or variable accretion (e.g., Jayawardhana et al. 2003; Natta et al. 2004).

c These targets were found to be active stars or variable accretors and are discussed in greater detail in Appendix A.1.

${ }^{\mathrm{d}}$ White et al. (2007) measured an RV of $-1.46 \pm 2.21 \mathrm{~km} \mathrm{~s}^{-1}$, consistent with our measurements.

e Note that SR21A shows $\mathrm{H} \alpha$ in absorption rather than in emission. See Appendix A.2 for further discussion of this object.

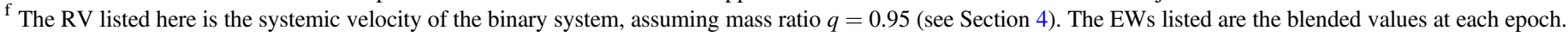

risk of confusing rotational broadening for thermal broadening, their $\mathrm{S} / \mathrm{N}$ were consistently too low for use.

Our results were sensitive to the assumed limb-darkening coefficient, $\epsilon$, with an $\sim 15 \mathrm{~km} \mathrm{~s}^{-1}$ deviation in $v \sin i$ for a change $\Delta \epsilon \sim 0.1$. Due to this sensitivity, we used the Claret (2000) linear limb-darkening coefficient catalog for each source, using characteristic values of $\log g$ and effective temperatures (Gizis 1997; Casagrande et al. 2008; Rajpurohit et al. 2013) for their literature SpTs (for $\mathrm{G}$ and $\mathrm{K}$ stars) and those determined by their TiO-7140 indices (for M stars; see Section 3.3).
For each observation, we took a $\mathrm{S} / \mathrm{N}$-weighted average of the $v \sin i$ as measured from the $\mathrm{Li}$ I and $\mathrm{Ca}$ I lines. For each target, we took the average of the per-observation measurements. Uncertainties were added in quadrature. Two targets in our sample have $v \sin i$ measurements reported in the literature: RX J1852.3-3700 (23.05 $\pm 3.59 \mathrm{~km} \mathrm{~s}^{-1}$ White et al. 2007); and SSTc2d J162740.3-242204 $\quad\left(14.7 \pm 0.9 \mathrm{~km} \mathrm{~s}^{-1}\right.$ Torres et al. 2006). Both values are within the uncertainties of our measured values. We present our measurements in Table 4. 
Table 3

Radial Velocity Standards

\begin{tabular}{lcr}
\hline \hline Name & Spectral Type & \multicolumn{1}{c}{$\begin{array}{c}\mathrm{RV}^{\mathrm{a}} \\
\mathrm{km} \mathrm{s}^{-1}\end{array}$} \\
\hline GJ 514 & M1 & $14.56 \pm 0.40$ \\
GJ 699 & M4 & $-110.51 \pm 0.40$ \\
GJ 908 & M2 & $-71.15 \pm 0.40$ \\
\hline
\end{tabular}

Note.

${ }^{a}$ RVs are taken from Nidever et al. (2002), who quote an RV stability of $0.05 \mathrm{~km} \mathrm{~s}^{-1}$. The zero point of the absolute RVs in this study is uncertain at the $0.4 \mathrm{~km} \mathrm{~s}^{-1}$ level (Marcy \& Benitz 1989).

Table 4

Spectral Types and Rotational Velocities

\begin{tabular}{|c|c|c|c|}
\hline Name & $\begin{array}{l}\text { SpT } \\
\text { (Lit.) }\end{array}$ & $\begin{array}{c}\text { SpT } \\
\text { (This Work) }\end{array}$ & $\begin{array}{c}v \sin i \\
\mathrm{~km} \mathrm{~s}^{-1}\end{array}$ \\
\hline SZ Cha & K0 & $\ldots$ & $34.0 \pm 0.4$ \\
\hline $\mathrm{T} 25$ & M2.5 & M1.4 \pm 0.6 & $24.3 \pm 0.4$ \\
\hline $\mathrm{T} 35$ & K8 & $\cdots$ & $29 \pm 3$ \\
\hline RX J1852.3-3700 & K7 & $\ldots$ & $19.5 \pm 0.5^{\mathrm{a}}$ \\
\hline CrA-4111 & M4.5 & M7.4 \pm 0.6 & $65 \pm 6$ \\
\hline G-49 & M4 & M5.2 \pm 0.6 & $43 \pm 4$ \\
\hline G-102 & M5 & $\mathrm{M} 7.6 \pm 0.6$ & $41 \pm 4$ \\
\hline SSTc2d J162118.5-225458 & M2 & M2.6 \pm 0.6 & $40 \pm 3$ \\
\hline SSTc2d J162218.5-232148 & K5 & $\cdots$ & $35 \pm 16$ \\
\hline SSTc2d J162245.4-243124 & M3 & $\mathrm{M} 2.1 \pm 0.6$ & $42.3 \pm 0.2$ \\
\hline SSTc2d J162309.2-241705 & M? & $\mathrm{K}$ ? & $45.0 \pm 0.9$ \\
\hline SSTc2d J162332.8-225847 & M5 & $\mathrm{M} 4.6 \pm 0.6$ & $49 \pm 4$ \\
\hline SSTc2d J162336.1-240221 & M5 & M3.6 \pm 1.1 & $64 \pm 5$ \\
\hline SSTc2d J162506.9-235050 & M3 & $\mathrm{M} 1.3 \pm 0.6$ & $46 \pm 6$ \\
\hline SSTc2d J162623.7-244314 & K5 & $\cdots$ & $10 \pm 1$ \\
\hline SSTc2d J162646.4-241160 & G5 & $\ldots$ & $49 \pm 5$ \\
\hline DoAr28 & $\mathrm{K}$ ? & $\cdots$ & $27 \pm 19$ \\
\hline SR21A & G3 & $\cdots$ & $65 \pm 3$ \\
\hline SSTc2d J162738.3-235732 & K5 & $\ldots$ & $42 \pm 2$ \\
\hline SSTc2d J162739.0-235818 & K6 & $\ldots$ & $43 \pm 23$ \\
\hline SSTc2d J162740.3-242204 & K5 & $\cdots$ & $18 \pm 2^{\mathrm{b}}$ \\
\hline SSTc2d J162802.6-235504 & M3 & $\mathrm{M} 4.0 \pm 1.1$ & $60 \pm 12$ \\
\hline SSTc2d J162821.5-242155 & M3 & $\mathrm{M} 1.5 \pm 0.6$ & $63 \pm 6$ \\
\hline SSTc2d J162854.1-244744 & M2 & $\mathrm{M} 0.3 \pm 0.6$ & $49 \pm 7$ \\
\hline SSTc2d J163020.0-233108 & M4 & $\mathrm{M} 2.4 \pm 0.6$ & $50 \pm 6$ \\
\hline SSTc2d J163033.9-242806 & M4 & $\mathrm{M} 4.7 \pm 0.6$ & $60 \pm 8$ \\
\hline SSTc2d J163145.4-244307 & M4 & $\mathrm{M} 2.3 \pm 1.3$ & $57 \pm 12$ \\
\hline SSTc2d J163154.4-250349 & M4 & $\mathrm{M} 2.5 \pm 0.7$ & $57 \pm 14$ \\
\hline SSTc2d J163154.7-250324A ${ }^{c}$ & K7 & $\mathrm{K} 7.0 \pm 0.5$ & $18.2 \pm 0.9$ \\
\hline SSTc2d J163154.7-250324B ${ }^{c}$ & $\ldots$ & $\mathrm{K} 9.0 \pm 0.5$ & $23 \pm 5$ \\
\hline SSTc2d J163205.5-250236 & M2 & M0.8 \pm 0.6 & $48 \pm 5$ \\
\hline SSTc2d J163355.6-244205 & K7 & $\cdots$ & $43 \pm 4$ \\
\hline
\end{tabular}

Notes.

${ }^{\text {a }}$ White et al. (2007) report a $v \sin i=23.05 \pm 3.59 \mathrm{~km} \mathrm{~s}^{-1}$ for this target, within $1 \sigma$ of our measured value.

${ }^{\mathrm{b}}$ Torres et al. (2006) report a $v \sin i=14.7 \pm 0.9 \mathrm{~km} \mathrm{~s}^{-1}$ for this target, within $2 \sigma$ of our measured value.

${ }^{c}$ This target is an SB2. In this Table we report the properties of each component. See Section 4.

\subsection{SpT Measurements}

M-dwarf spectra are dominated by $\mathrm{TiO}$ molecular bands. Wilking et al. (2005) defined the TiO-7140 index as a way of determining the SpTs of M-dwarfs: a ratio of the mean flux of two $50 \AA$ bands centered on $7035 \AA$ (the continuum band) and

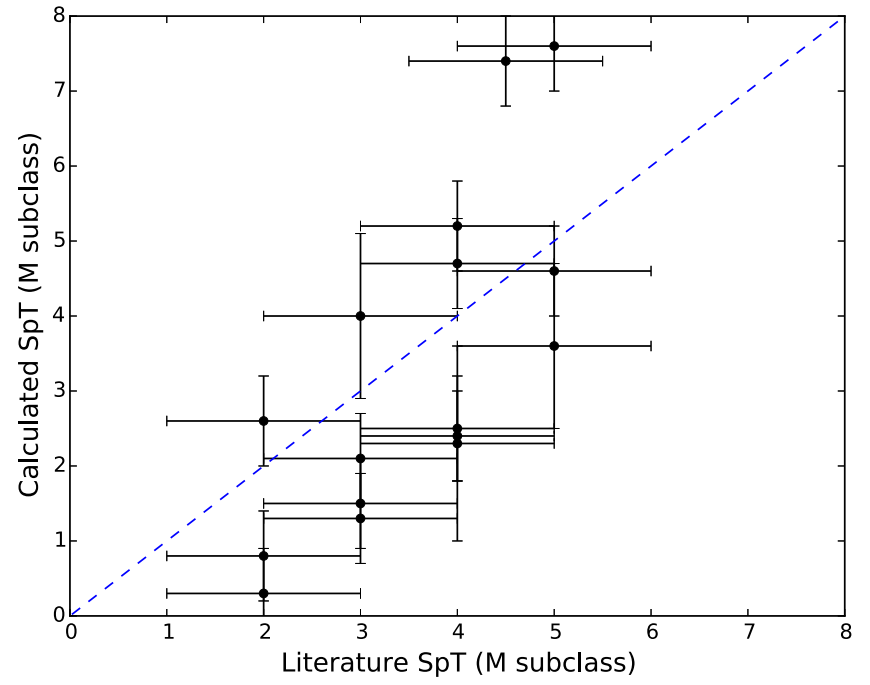

Figure 3. M-subclasses of targets reported as M-dwarfs in the literature (i.e., $0 \rightarrow \mathrm{M} 0,1 \rightarrow \mathrm{M} 1$, etc.), with their literature subclasses compared with our own measurements based on their TiO-7140 indicies. A 1:1 relation is overlaid in blue. Our measurements are broadly consistent with the literature values. Larger deviations from the literature values at subclasses $\geqslant 5$ occur because the TiO-7140 index is less well-constrained for late M SpTs.

$7140 \AA$ (the $\mathrm{TiO}$ band). We used the calibration given in Shkolnik et al. (2009)

$$
\mathrm{SpT}=(\mathrm{TiO}-7140-1.0911) / 0.1755
$$

to measure the SpTs of all of our targets. Strictly, this relation holds for SpTs M0-M5, which covers the range of SpT literature values of M-dwarfs in our sample. ${ }^{9}$ Our results are shown in Figure 3. The error bars for literature values are \pm 1 subclass, largely from C10. The error bars for our own measurements are the root mean square $=0.6$ scatter of the Shkolnik et al. (2009) calibration, added in quadrature with the standard deviation of the measured SpTs across the multiple epochs each target was observed. They favor a linear fit of

$$
\mathrm{SpT}_{\text {This work }}=(1.5 \pm 0.4) \mathrm{SpT}_{\text {Lit. }}+(-2 \pm 1),
$$

i.e., we measure SpTs consistent with the literature values to $\sim \pm 1 \mathrm{SpT}$ subclass. Our measured SpT per target is shown in Table 4 along with the literature values (see references in Table 1) and $v \sin i$ (see above). We do not revise K-type SpTs with the exception of SSTc2d J163154.7-250324, which we find to be an SB2 (see Section 4). For this system we report the derived SpTs and $v \sin i$ for each component. The lack of TiO in the spectra of the two G stars in our sample (SSTc2d J162646.4-241160 and SR21A) indicates that they are hotter than $\mathrm{M}$ stars, but the difference between very young $\mathrm{G}$ and $\mathrm{K}$ stars is not well-constrained (e.g., Beuther et al. 2014) and therefore we do not revise their SpTs.

\section{SPECTROSCOPIC BINARY DETECTION}

Single-lined SBs, SB1s are revealed by significant RV variability between observations. We performed $\chi^{2}$ tests on the RVs of targets with single-peaked cross-correlation functions

\footnotetext{
9 An interesting case is that of the target SSTc2d J162309.2-241705, for which C10 report an uncertain SpT of "M?." We measure a negative $\mathrm{M}$-subclass for this target, suggesting that it may be a late $\mathrm{K}$.
} 




Figure 4. Spectrum of SSTc2d J163154.7-250324 on UT110614. The SB2 nature of the system is evident in all absorption features, including the Li lines at $6708 \AA$.

(CCFs) using the target's average RV as a flat prior and found no SB1s in our sample of 31 TD objects. As mentioned above, the fact that all of the RVs agree with both the sample average for their SFR and the association average from the literature argues against any of our targets being long-period SB1s, or $P \sim 1$ year systems whose RV was serendipitously measured by us at the same orbital phase each year.

Cross-correlation of the target spectra with an RV standard spectrum reveals whether a star is an SB2 if the orbital phase at the time of observation allows resolvable RV motion such that CCF is double-peaked. We found one SB2 in the sample (SSTc2d J163154.7-250324), which we discuss in the subsections below.

\subsection{Measured Properties of SSTc2d J163154.7-250324}

The stellar features were blended enough on UT100620 and UT100622 that we could not confirm it as an SB2 using those observations alone. SSTc2d J163154.7-250324 clearly exhibited a double-peaked CCF one year later on UT110614. We measured the RVs of the primary and secondary components to be $16 \pm 2 \mathrm{~km} \mathrm{~s}^{-1}$ and $-27 \pm 2 \mathrm{~km} \mathrm{~s}^{-1}$, respectively. This gives a systemic RV of $\gamma=-5.4 \pm 0.5 \mathrm{~km} \mathrm{~s}^{-1}$ based on fluxes in the two CCF peaks (flux ratio of $0.68 \pm 0.07$; see below). We measured values of $v \sin i$ for the primary and secondary as $18.2 \pm 0.9 \mathrm{~km} \mathrm{~s}^{-1}$ and $23 \pm 5 \mathrm{~km} \mathrm{~s}^{-1}$, respectively, following the method described in Section 3.2 on the unblended absorption lines seen on UT110614. A section of the stellar spectrum and the CCFs of this SB2 are shown in Figures 4 and 5 respectively.

Since we resolved the two CCF peaks, we were able to estimate SpTs and component masses of the individual stars. Assuming a flux-weighted relation (where $f_{i}$ is the integrated flux of Gaussian fits to the cross-correlation peaks at $\mathrm{R}$ band wavelengths; Figure 5) between component and integrated SpTs (Cruz \& Reid 2002; Reid \& Cruz 2002; Daemgen et al. 2007) for primary star $A$ and secondary $B$ :

$$
\operatorname{SpT}_{\text {int }}=\left(f_{A} \operatorname{SpT}_{A}+f_{B} \operatorname{SpT}_{B}\right) /\left(f_{A}+f_{B}\right) .
$$

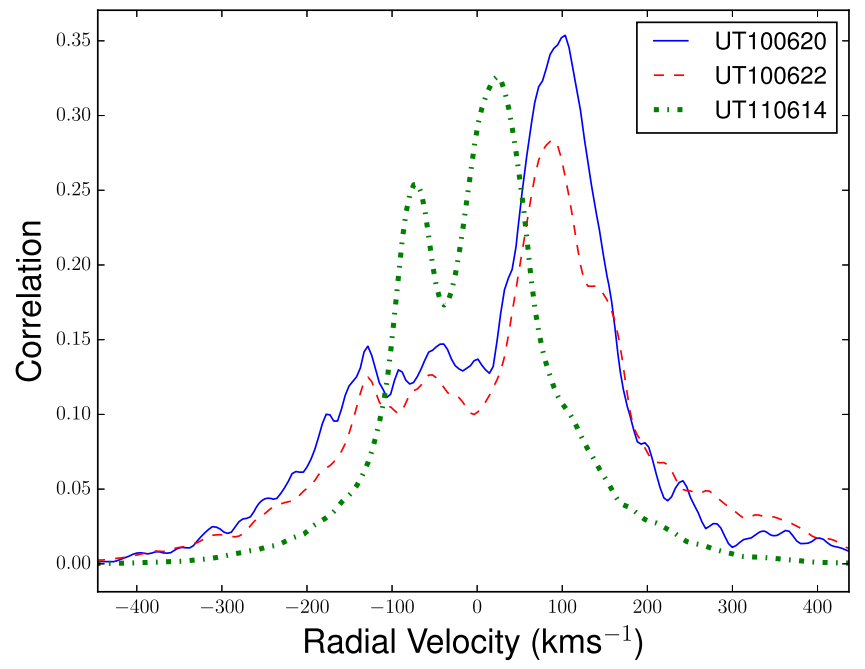

Figure 5. CCFs of SSTc2d J163154.7-250324 averaged across apertures used. Spectra taken in 2010 were cross-correlated against GJ 699 and those in 2011 against GJ 514.

Table 5

Properties of the SSTc2d J163154.7-250324 System

\begin{tabular}{lccc}
\hline \hline Property & System & A & B \\
\hline SpT & $\mathrm{K} 7^{\mathrm{a}}$ & $\mathrm{K} 7.0 \pm 0.5$ & $\mathrm{~K} 9.0 \pm 0.5$ \\
$\mathrm{RV}\left(\mathrm{km} \mathrm{s}^{-1}\right)$ & $-5.4 \pm 0.5$ & $16 \pm 2$ & $-27 \pm 2$ \\
$v \sin i\left(\mathrm{~km} \mathrm{~s}^{-1}\right)$ & $\ldots$ & $18.2 \pm 0.9$ & $23 \pm 5$ \\
Separation $(\mathrm{au})$ & $<0.6$ & $\ldots$ & $\ldots$ \\
Period $($ days & $<150$ & $\ldots$ & $\ldots$ \\
Mass ratio & 0.95 & $\ldots$ & $\ldots$ \\
\hline
\end{tabular}

Note.

${ }^{a}$ Cieza et al. (2010).

Using the Kepler's Third law we related component masses $M_{n}$ to velocity amplitudes $K_{n}$ :

$$
M_{A} / M_{B}=K_{A} / K_{B}
$$

We imposed a limit on $\mathrm{SpT}$ and magnitude (Daemgen et al. 2007; Shkolnik et al. 2010):

$$
\Delta R=M_{R}\left(\mathrm{SpT}_{B}\right)-M_{R}\left(\mathrm{SpT}_{A}\right) .
$$

We measure a flux ratio of $0.68 \pm 0.07$ for the CCF peaks. Thus, we determine that SSTc2d J163154.7-250324 is composed of a $\mathrm{K} 7( \pm 0.5)$ and a $\mathrm{K} 9( \pm 0.5)$ star, with mass ratio $q \simeq 0.95$. Using Kepler's Third Law, we calculated the orbital limits to be $a<0.6$ au and period $P<150$ days. Our measurements of this system are summarized in Table 5.

\subsection{Comparison to the Literature}

Before the SB2 SSTc2d J163154.7-250324 was identified as a TD, it had already been measured to have an infrared excess in its SED by 2MASS and exhibit the visible spectral properties of a YSO (Ratzka et al. 2005, who refer to the system as $\mathrm{H} \alpha 74$ and/or ISO-Oph 207). The disk is unresolved in the CHARM2 catalog (Richichi et al. 2005). Padgett et al. (2008) reported the YSO to reside in the most populated region of the Oph SFR. Evans et al. (2009) reported J163154.7-250324 to have an extinction-corrected temperature and a luminosity of $3100 \mathrm{~K}$ and $2.5 L_{\odot}$, respectively. It is listed as a YSO candidate star with extinction from dust, but no evidence of local extinction 
from a surrounding envelope. Cieza et al. (2009), like Ratzka et al. (2005), found no evidence for multiplicity.

C10 identified the system as a TD and determined it to be a K7-type star, in agreement with our measurements above. Their $\mathrm{Li}$ I measurements were too low in $\mathrm{S} / \mathrm{N}$ and therefore unreported, so we cannot compare them to our own measurements of $0.5 \pm 0.2 \AA$. They detected strong emission from the $\mathrm{Ca}$ II triplet, providing further evidence that it is indeed a PMS object. Their measurement of the $\mathrm{H} \alpha 10 \%$ velocity-width $\left(470 \mathrm{~km} \mathrm{~s}^{-1}\right)$ is similar to our own $\left(364 \pm 61 \mathrm{~km} \mathrm{~s}^{-1}\right)$, but variation is expected due to variable accretion and the SB2 nature of the object. C10 found no evidence for a companion, as their study was not sensitive to separations $<8$ au. They derive a limit on disk mass of $M_{D}<1.1 M_{\text {Jup }}$ and classify it as a grain growth-dominated TD (as mentioned in Section 1, this is their most common TD subclass).

\section{SAMPLE SENSITIVITY TO COMPANIONS}

We proceeded in a completeness study of our results following Section 6.1 of Duquennoy \& Mayor (1991) and Section 3.1 of Melo (2003), considering the observation cadence for each target, $v \sin i$, and the RV uncertainties of each spectrum. We sought to constrain how sensitive we were to SB1s within each TD for different orbital periods and secondary masses.

We estimated the primary mass of each target based on its absolute magnitude using low-mass solar metallicity stellar evolution models from Baraffe et al. (2015). To estimate absolute magnitudes we apply the distance to Oph of $140 \mathrm{pc}$, for Cha targets we used a distance of $160 \mathrm{pc}$ (Feigelson \& Lawson 2004), and for CrA and Cor targets we use a distance of 140pc (Sicilia-Aguilar et al. 2008). We use the Baraffe et al. (2015) 1 Myr CFHT tracks for all targets (e.g., Furlan et al. 2009; Kim et al. 2009). The 2MASS $J$ magnitudes, available for all of our targets (and $K$ where available), coupled with these age and distance estimates allowed us to estimate the mass of each target. We did not correct for extinction; NIR magnitudes are the least affected by optical- and NIR-excesses from accretion and inner disks. The average change in mass estimate between the Baraffe et al. (1998) and Baraffe et al. (2015) models was $|\Delta M|=0.2 M_{\odot}$. Such a difference has little impact on our results.

With these estimated primary masses of each target, we generated $10^{6}$ artificial companions, each with mass ratio $(q)$, period $(P)$, time of periastron, inclination $(i)$, and longitude of periastron $(\omega)$ chosen randomly from a uniform distribution. Eccentricity $(e)$ was chosen to be zero for $P<8$ days, and drawn from the Hyades distribution of Burki \& Mayor (1986) for $P>8$ days. The solution of Kepler's Equation followed Chapter 2.5 of Hilditch (2001). At each date of observation, we calculated the RV for each artificial companion and added to this a random uncertainty drawn from a Gaussian distribution centered around our measured uncertainty of the (real) primary RV (Table 2).

To avoid an overestimate of detection probability at high mass ratios and long orbital periods (where we expect SB2 systems to lie in this $\left(M_{\text {secondary }}, P\right)$-space) we calculated the flux-weighted systemic velocity of the binary system

$$
\gamma=\frac{\mathrm{RV}_{1} A_{1}+\mathrm{RV}_{2} A_{2}}{A_{1}+A_{2}}
$$

where $A_{2} / A_{1}$ is the flux ratio of the stars, and $\mathrm{RV}_{1}$ and $\mathrm{RV}$ are the RVs of each star. Their orbits are related by angle $\theta_{2}=\theta_{1}+\pi$, orbiting the center of mass. We interpolated the fluxes in the I-band using the Baraffe et al. (2015) 1 Myr CFHT tracks. Defining $\quad \alpha_{1}=\cos \left(\theta_{1}+\omega\right)+e \cos \omega$, $\alpha_{2}=\cos \left(\theta_{1}+\pi+\omega\right)+e \cos \omega$, and expressing the flux ratio as $F_{R}=A_{2} / A_{1}$, the systemic $\mathrm{RV}$ of the system is then

$$
\gamma=\frac{2 \pi a \sin i}{\left(1+F_{R}\right) P\left(1-e^{2}\right)^{1 / 2}}\left(M_{2} \alpha_{1}+M_{1} \alpha_{2} F_{R}\right) .
$$

We then tested if the artificial companion could be "detected" by whether the root mean square of the values of $\gamma$ (each given by Equation (7)) was greater than $0.7 \mathrm{~km} \mathrm{~s}^{-1}$, i.e., greater than the upper limit on the standard errors of our nonSB RVs. With over $10^{6}$ simulations per target, we were able to map probabilities of detection in $\left(M_{\text {secondary }}, P\right)$-space. The results of these simulations are shown in Figure 6, which shows contour levels in $10 \%$ bins from $40 \%$ to $100 \%$ chance of detection. ${ }^{10}$ The periodic modulation in the contour patterns demonstrates our insensitivity to orbital periods that are multiples of our observing cadence.

The results of these simulations allow us to accurately assess our sensitivity to companions on a per-target basis, with a degeneracy in $P$ and mass ratio $q .{ }^{11}$

For example, for SZ Cha we are sensitive to ( $\geqslant 80 \%$ chance of detection) a $0.4 M_{\odot}$ companion at periods $P<30$ days and $70<P<75$ days, whereas for SSTc2d J162623.7-244314 we are sensitive to a $0.4 M_{\odot}$ companion at all periods. Such an example illustrates the challenge in quoting an "overall" or "average" sensitivity for this non-homogeneous sample. We are, however, consistently sensitive to short-period $(P<\sim 100$ days) SBs for all of our targets.

\section{DISCUSSION}

Prato (2007) measures a spectroscopic binary fraction of $0.12_{-0.04}^{+0.08}$ among Oph K7-M4 stars. Of the 33 targets in Prato (2007), two are TD objects but neither appears to be an SB: ROXR1 20 (C10) and RX J1614.4-1857 (Wahhaj et al. 2010). Of the five SBs they found, one hosts a debris disk (RX J1612.6-1924, Wahhaj et al. 2010) and the rest are diskless (RX J1612.3-1909, RX J1622.7-2325, and RX J1622.8-2333; Wahhaj et al. 2010; ROXR1 14 Cieza et al. 2007; Rosero et al. 2011). We found one SB2 in a sample of 24 Oph TD objects, and no SBs among the TD objects in the other SFRs surveyed. Including the two TD targets from Prato (2007) in our Oph sample, we find that multiplicity among TD objects in this region to be $1 / 26$. We measure an SB fraction of $0.04_{-0.03}^{+0.12}$, determining uncertainties following the binomial theorem. This is consistent with that of the non-TD, late-type stars in Ophiuchus (Prato 2007) and the young stars in Chameleon and Taurus-Auriga $\left(0.07_{-0.03}^{+0.05}\right.$ and $0.06_{-0.02}^{+0.03}$, respectively; mass range $0.2-3 M_{\odot}$; Nguyen et al. 2009). The result is also in agreement with Lodieu et al. (2014), who report an SB fraction of $0.054 \pm 0.038$ for wide binaries $(50-150 \mathrm{au})$ in which one star is a planet-host. Their SB fraction for wide, planet-hosting substellar binaries is also within our range at $0.027 \pm 0.027$.

\footnotetext{
${ }^{10}$ While contour levels run between these values, they rarely go below a $60 \%$ chance of detection. This happens only for the lowest secondary masses.

${ }^{11}$ One can convert between limits on the period and limits on the semimajor axis of separation $a$ using the relation $a\left(P, q, M_{\text {primary }}\right)=$ $(P / 2 \pi)^{2 / 3}\left(G(1+q) M_{\text {primary }}\right)^{1 / 3}$.
} 

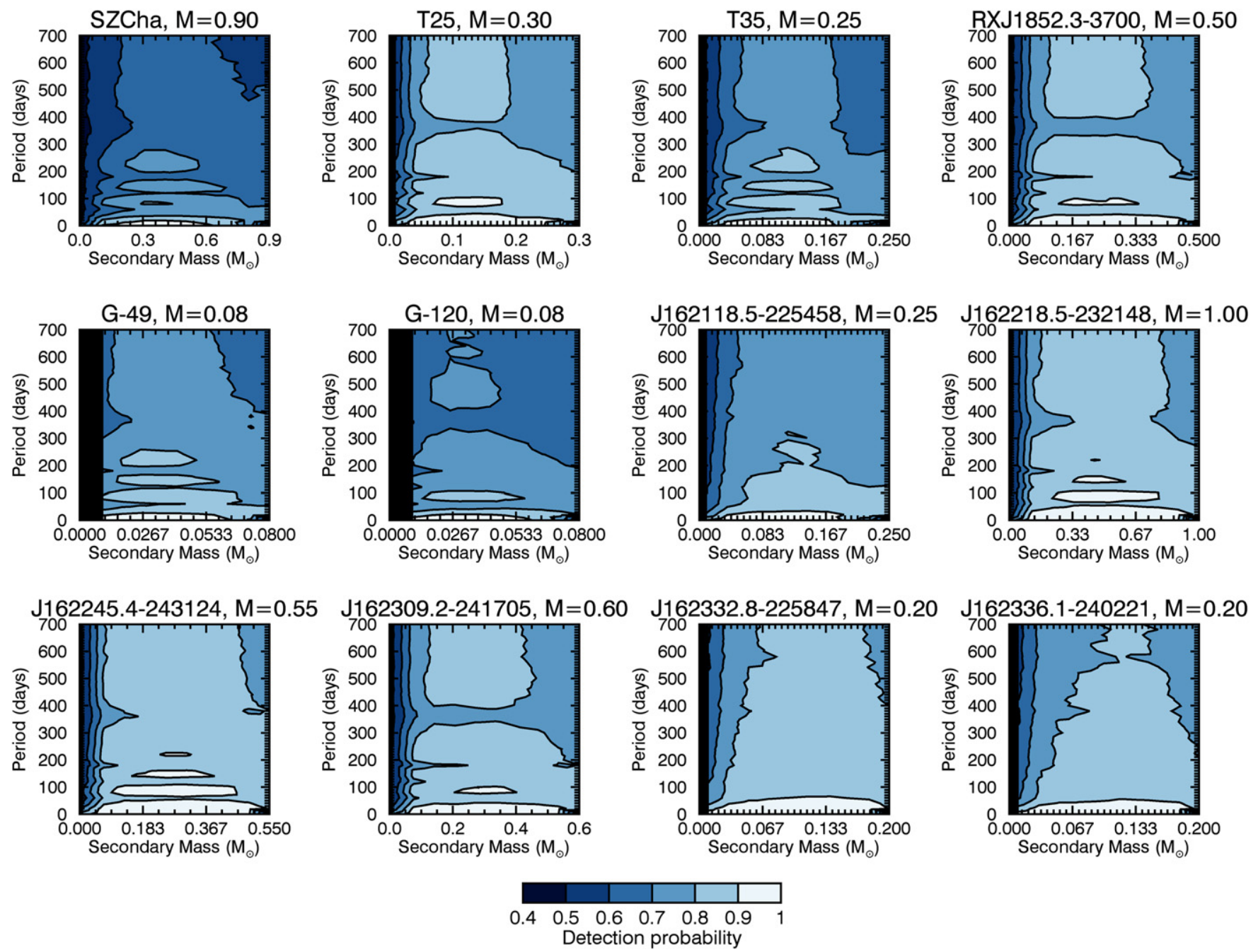

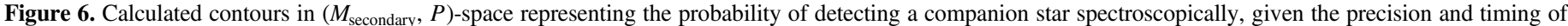

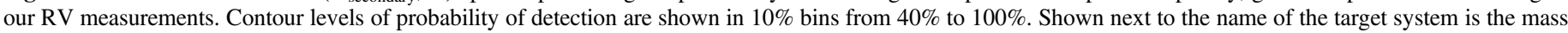

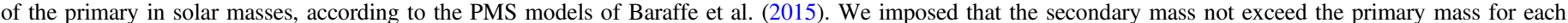

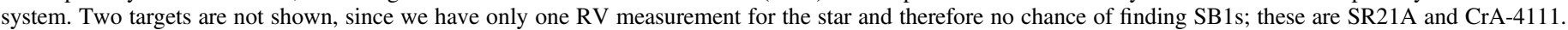
For the Ophiuchus targets we have dropped the "SSTc2d" prefix.

We also conclude that there is no significant difference between our result and that among the low-mass $\left(0.08 M_{\odot}-0.6 M_{\odot}\right)$ field stars in the surveys of Duquennoy \& Mayor (1991) and Fischer \& Marcy (1992), $0.09_{-0.02}^{+0.03}$ and $0.03_{-0.02}^{+0.04}$, respectively. Likewise, Raghavan et al. (2010) found an SB fraction of $0.073_{-0.012}^{+0.014}$ among nearby old solar-type dwarfs.

That the fraction of SBs in TDs is similar to diskless stars (within uncertainties) may suggest that whatever causes the disk dissipation is independent of binarity, i.e., disks around close SBs evolve on a similar timescale as those around single stars. Such a conclusion would mean that planet formation timescales around SBs may be comparable to those around single stars.

However, several studies (Ghez et al. 1997; White \& Ghez 2001; Cieza et al. 2009; Duchêne 2010; Kraus et al. 2012) have found protoplanetary disk fractions are lower for young ( $\leqslant 5 \mathrm{Myr}$ ) binary systems with $a<50$ au than for single stars or wider binaries. With the exception of Kraus et al. (2012), these studies were not sensitive to the tight binary systems to which our study was especially sensitive. Kraus et al. (2012) and Cheetham et al. (2015), sensitive to similar binary separations to our study, find the disk fraction among close visual binaries $(0.1-40 \mathrm{au})$ in Taurus-Auriga and Ophiuchus to be lower than for single stars. Their studies did not distinguish between circumbinary TDs and other disk types (e.g., debris disks). If the SB fraction of TD systems is actually lower than that in the diskless population, this suggests that the binary TD stage of a close-in binary is even shorter than the TD stage of single stars.

While we have searched for SBs specifically in TDs, Prato (2007) made a complementary search for SBs irrespective of knowledge of their disks. None of the SBs she found were in transitional or protoplanetary disks. This is consistent with SBs having a faster TD phase and that the overall disk fraction among SBs remains low down to small separations.

Such a conclusion would be in contention with the results of Alexander (2012), whose models suggest that protoplanetary disks around tight binaries $(a \leqslant 1 \mathrm{au})$ are longer-lived than those around wider binaries. This is because the very close binaries efficiently clear the inner disk to radii larger the 

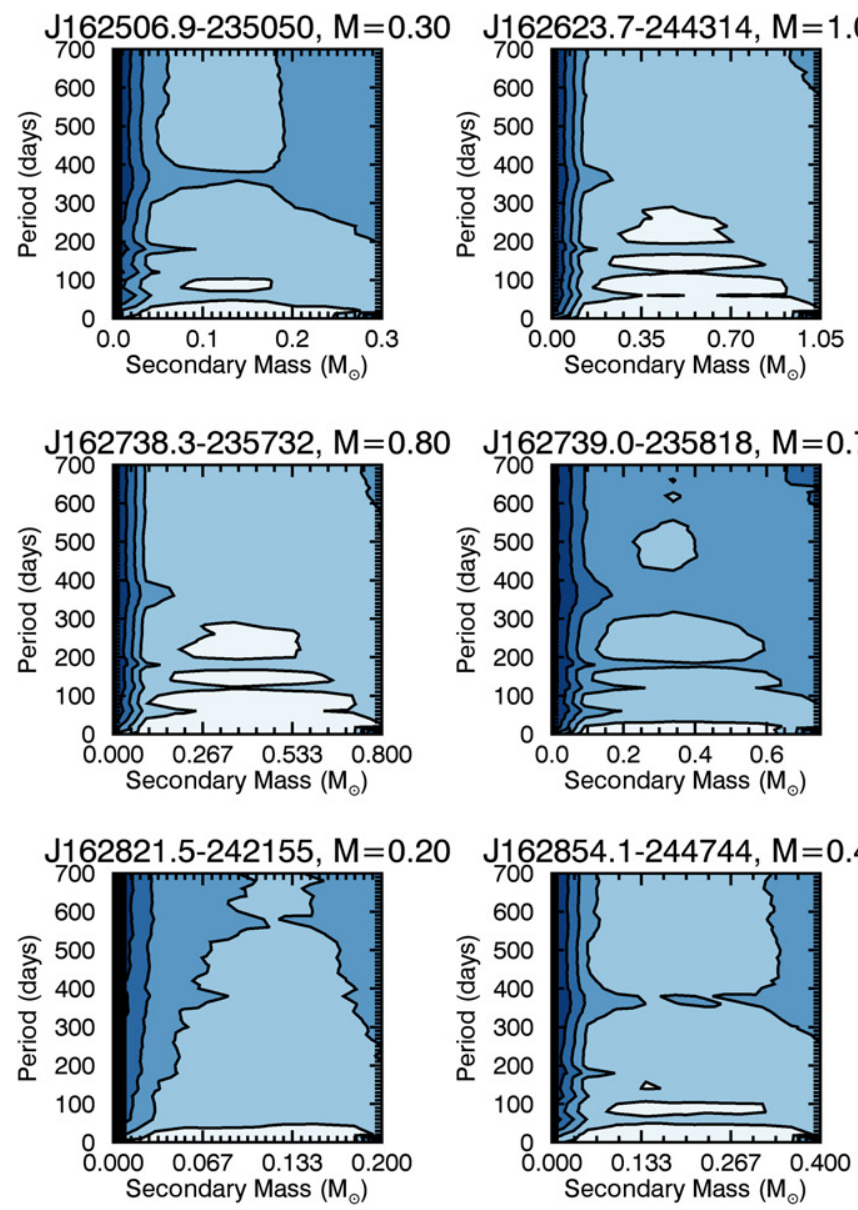
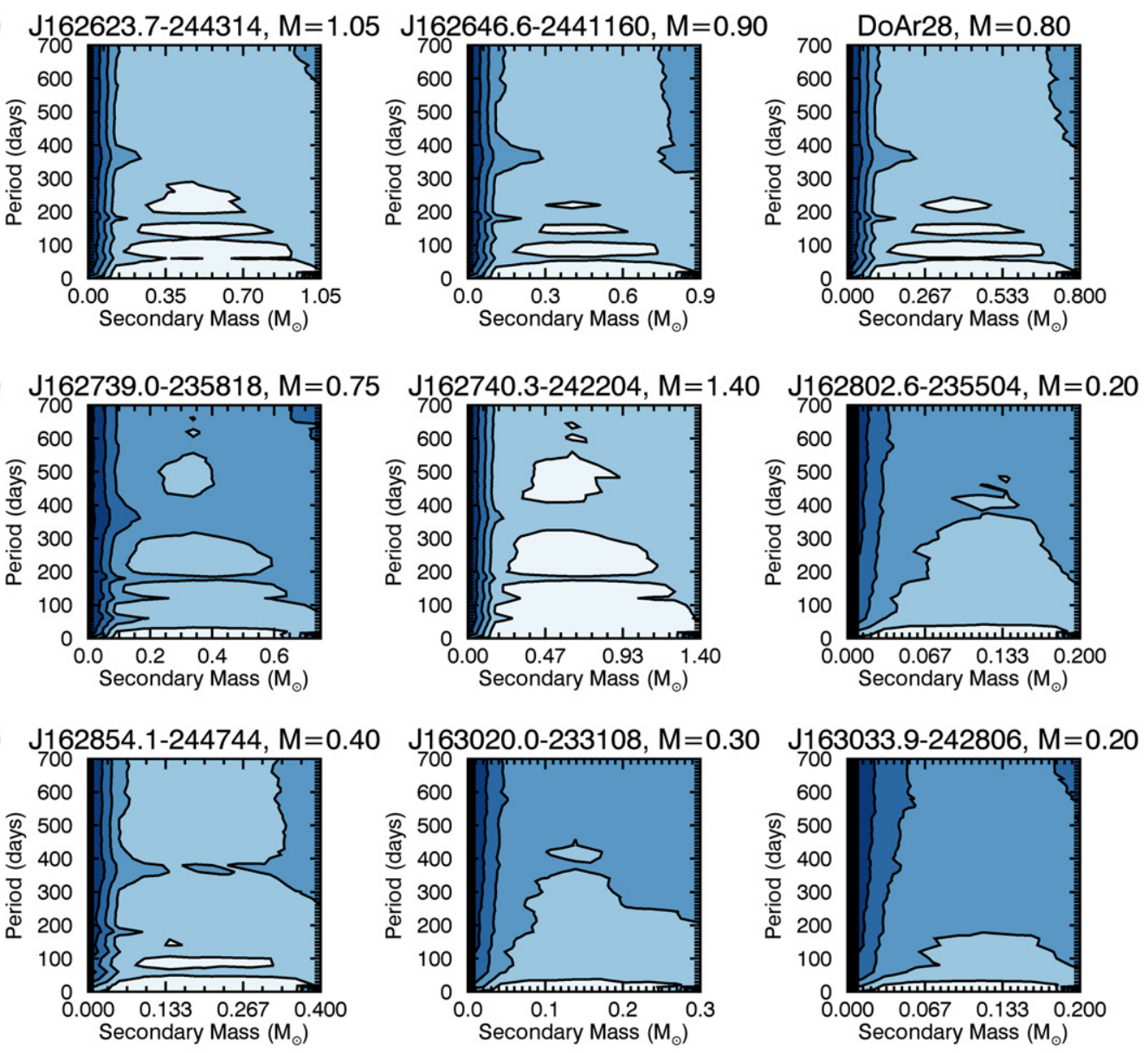

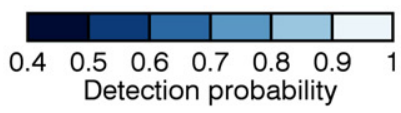

Figure 6. (Continued.)

characteristic radius of photoevaporative winds, suppressing total disk dispersal.

That we only find one SB in our TD sample could reflect that dissipation is so fast among these objects that most disks are completely dispersed by the age of Oph. However, we do not have the statistics to draw a steadfast conclusion. But since we find no evidence that TD objects have higher SB fractions than other stellar populations, we can infer that close-in binaries are not likely the primary cause of inner holes in TDs. This leads to giant planet formation as a more likely explanation.

\section{SUMMARY}

We have presented a spectroscopic survey of 31 TD stars, 24 of which lie in Ophiuchus. We found one of the Oph stars to be an SB2 (SSTc2d J163154.7-250324). This system is composed of a $\mathrm{K} 7( \pm 0.5)$ and a $\mathrm{K} 9( \pm 0.5)$ star, with mass ratio $q \simeq 0.95$ and orbital limits $a<0.6$ au and $P<150$ days. The average $\mathrm{RV}$ of our Oph targets is $-6.6 \mathrm{~km} \mathrm{~s}^{-1}$ with a dispersion of $1.3 \mathrm{~km} \mathrm{~s}^{-1}$. The median uncertainty in the measured RVs including systematic uncertainties is $0.4 \mathrm{~km} \mathrm{~s}^{-1}$. The average $\mathrm{RVs}$ of all four clouds surveyed (Cha, Cor, $\mathrm{CrA}$ and $\mathrm{Oph}$ ) are consistent with the literature values. With this single SB detection, we measure an SB fraction of $0.04_{-0.03}^{+0.12}$. This finding is consistent with that of non-TD late-type stars in and outside of the region. This suggests that a TD may not be dispersed more efficiently by a tight binary than by a single star, and may imply that planet formation timescales around close binaries are comparable to those around single stars.

We thank M. Hughes for her contribution of RX J1852.3-3700 to the target list prior to its publication; L. Prato, C. Johns-Krull, and Cullen H. Blake for helpful discussions; and the anonymous referee for her/his insightful comments. S. A. Kohn acknowledges the support of NSF REU grant AST1004107 through Northern Arizona University and Lowell Observatory. J. Llama acknowledges support from NASA Origins of the Solar System grant No. NNX13AH79G and from STFC grant ST/M001296/1. This research made use of the SIMBAD database, operated at CDS, Strasbourg, France.

Facility: LCO: Magellan (MIKE).

\section{APPENDIX}

We present here the accretion properties and $\mathrm{H} \alpha$ and $\mathrm{Li}$ I measurements of individual stars. 

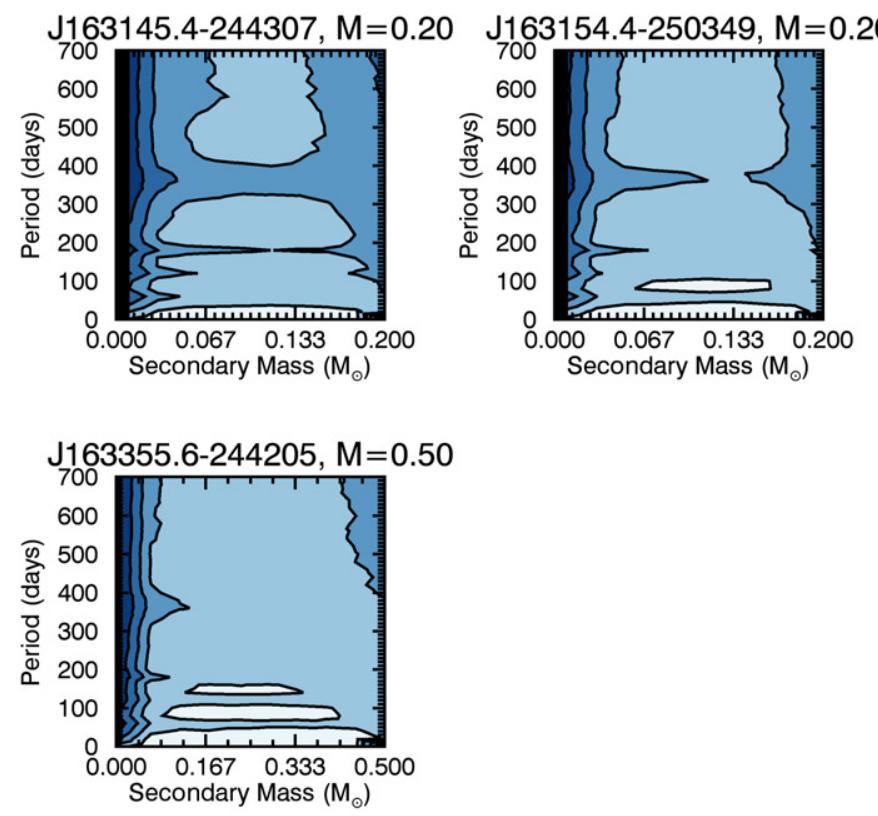
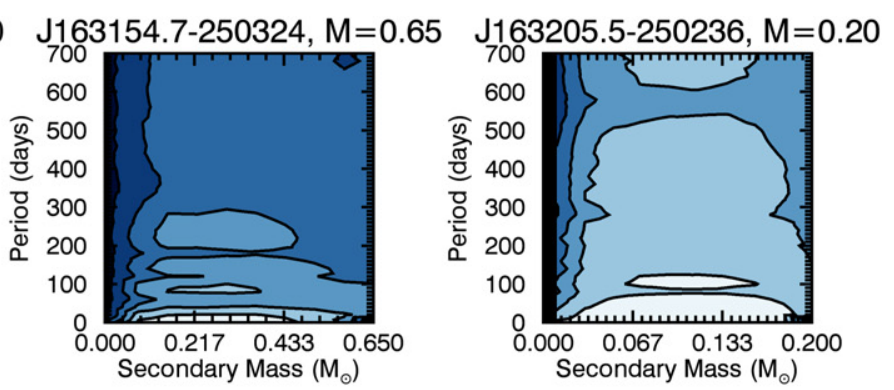

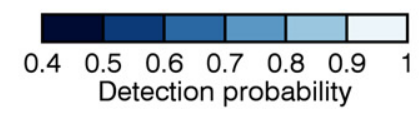

Figure 6. (Continued.)

\section{A.1. Age and Accretion Diagnostics}

The strength of the $\operatorname{Li}$ I line $(\lambda=6708 \AA)$ is a standard diagnostic for the age of low-mass stars, since lithium is rapidly depleted in their atmospheres. All but one of our targets exhibit $\mathrm{Li}$ I absorption, so we are only able to place upper limits on the absorption in the spectra of CrA-4111 due to low $\mathrm{S} / \mathrm{N}$ in our single observation of the system. The $\mathrm{Li}$ I should be undetectable in early $M$ stars after $\sim 20 \mathrm{Myr}$ (Baraffe et al. 1998; White \& Hillenbrand 2005). For G and K stars the depletion can take much longer $(\geqslant 300 \mathrm{Myr}$, e.g., Zickgraf et al. 2005). The age of Oph is $<10 \mathrm{Myr}$ (Duncan 1981; Chabrier et al. 1996; Martin et al. 1999a, 1999b; Zuckerman \& Song 2004, C10), and thus, all stars are expected to display $\mathrm{Li}$ absorption. For the 12 stars at sufficient $\mathrm{S} / \mathrm{N}$ to detect $\mathrm{Li}$ that were also observed in C10, our EWs are consistent with theirs. Our Li I EW measurements of DoAr 28 and SR21A are consistent with those of Magazzu et al. (1992) and James et al. (2006), respectively. We are not aware of any previous measurements of Li I in SSTc2d J162309.2-241705. The Li I EWs are listed in Table 2.

$\mathrm{H} \alpha(\lambda=6563 \AA)$ is an indicator of stellar activity and gas accretion onto a star. Applying the relation found by Natta et al. (2004), we use the $10 \%$ velocity-width (White \& Basri 2003) of the $\mathrm{H} \alpha$ emission to estimate the rate of accretion from the disk onto the star for targets with $\mathrm{H} \alpha 10 \%$ velocity-widths greater than $200 \mathrm{kmbs}^{-1}$ (this is true for 21 of our 31 targets). We find that of the 15 targets that were observed by $\mathrm{C} 10$ and had sufficient $\mathrm{S} / \mathrm{N}$ at $\mathrm{H} \alpha, 10$ have $10 \%$ velocity-widths within $3 \sigma$ of those measured by $\mathrm{C} 10$. The large variations in the remaining measurements are likely due to variable accretion upon the star (e.g., Nguyen et al. 2009). Six of our targets exhibited varying $\mathrm{H} \alpha$ during our observations as shown in Figure 7. These are SZ Cha, T25, SSTc2d J162118-225458， SSTc2d J162218-232148， SSTc2d
J162739-235818, and SSTc2d J162506-235050. The level of variability is unique for each target, but on average the EW varies by $\sim 80 \%$.

\section{A.2. SR21A}

At about 1-3 Myr old (which we note is older than the average age of Oph stars; Siess et al. 2000; Andrews et al. 2009; Brown et al. 2009; Follette et al. 2013), SR21A is the primary component of a wide binary system in Oph (Barsony et al. 2005). Its SpT has been reported to be an early G (Suarez et al. 2006; Andrews \& Williams 2007b), an early K (Struve \& Rudkjobing 1949), and an M star (Kimeswenger et al. 2004). Our measurements of its TiO7140 index (see Section 3.3) suggest it is not an M-type star $\left(\mathrm{SpT}_{\mathrm{TiO}}<0\right)$. SR21A displays $\mathrm{H} \alpha$ in absorption rather than in emission, ${ }^{12}$ the only such star in our sample. Li I absorption is also weaker than all of our other Oph objects. The $\mathrm{H} \alpha$ absorption could be due to variable accretion (Johns-Krull \& Valenti 2001; Baraffe et al. 2012; Hamilton et al. 2012). This is suggested by the broadened blue shoulder of the absorption (a feature possibly hidden by the $\mathrm{N}_{\text {II }}$ absorption on the red shoulder), a sign of possible accretion emission superimposing upon the photosphere absorption (Figure 8, left). Weak Li I absorption $(0.13 \pm 0.02 \AA$; Figure 8 , right $)$ might suggest that SR21A is older than expected ( $<10$ Myr; Chabrier et al. 1996), but the existence of a TD is evidence against the star being $>15$ Myr (Mamajek 2009; Muzerolle et al. 2010). While James et al. (2006) find a slightly higher EW for $\mathrm{Li}_{\mathrm{I}}$ in SR21A $(0.275 \pm 0.028 \AA$ versus our value of $0.13 \pm 0.02 \AA)$, they also note its inconsistency with other EWs in Oph. It is possible that the same episodic accretion that could be causing the

\footnotetext{
${ }^{12}$ It should be noted that SR21A is listed as an emission star in the SIMBAD database, but we did not observe is as such. Struve (1949) do list it as a nonemitting star in their spectral emission study of Oph.
} 

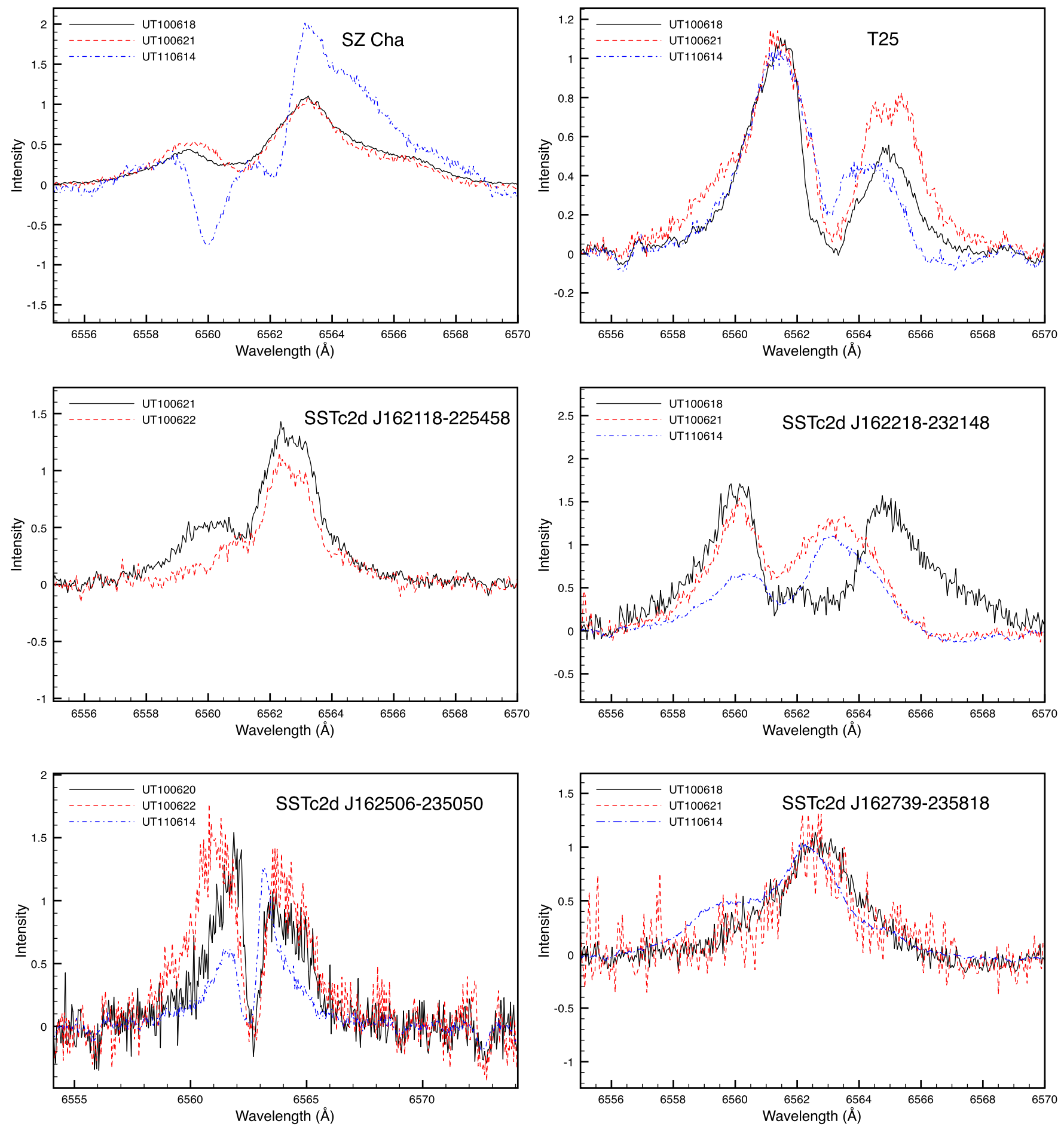

Figure 7. Variable accretion shown in $\mathrm{H} \alpha$.

$\mathrm{H} \alpha$ absorption has also resulted in abnormal Li depletion in this star (Baraffe et al. 2009, 2012; Baraffe \& Chabrier 2010). Depletion is also more realistic than the feature being veiled by accretion, since a disk hot enough for veiling would have caused SR21A to be disqualified as a TD object. However, we compared the EWs/line strengths of all of the lines in the same echelle order as the Li line in SR21A with the late K- and early M-type stars we observed on the same night (UT110614). We found that SR21A had weaker absorption for all lines, suggesting that veiling may still play a role. Overall weaker lines might instead indicate a lower overall metallicity compared with the other Oph targets.

An extensive study of the disk morphology of SR21A was recently presented by Follette et al. (2013). Their results may support the postulate of a substellar companion present within the SR21A disk at $\sim 18$ au from the primary (Eisner et al. 2009). Since we have just one observation of this system, we can neither confirm nor exclude this finding. 

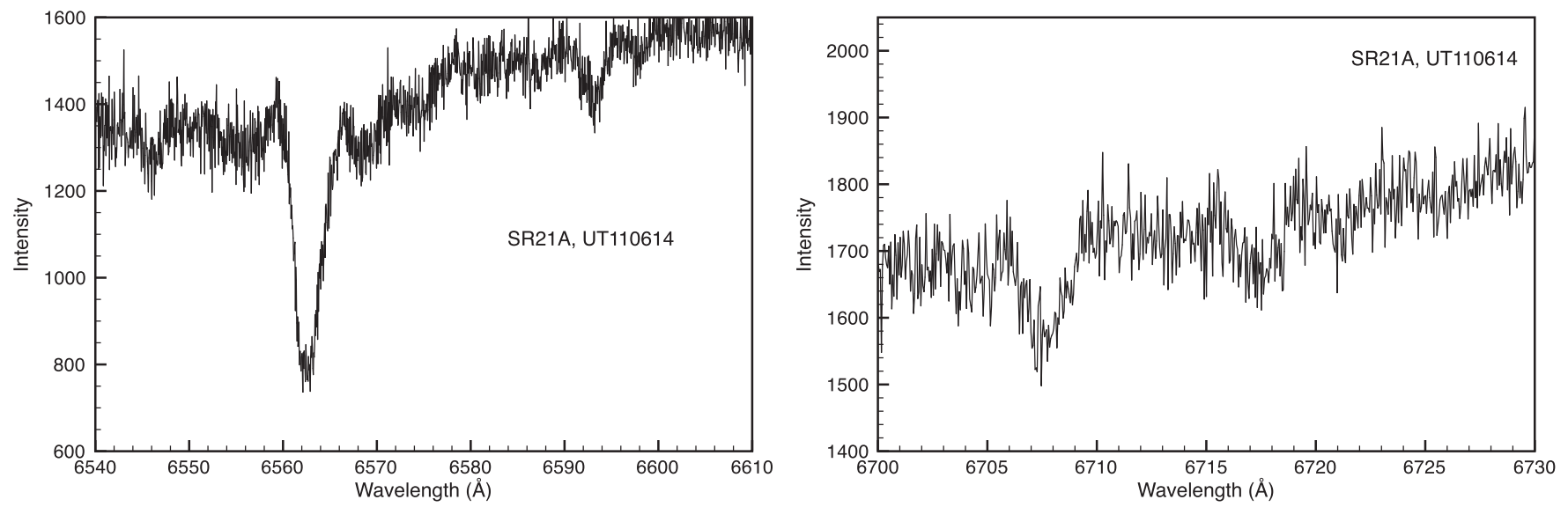

Figure 8. Spectral features of SR21A: H $\alpha$ absorption (left; $\mathrm{EW}=1.13 \pm 0.15 \AA$ ) and weak Li I absorption (right; $\mathrm{EW}=0.13 \pm 0.02 \AA$ ).

\section{REFERENCES}

Alexander, R. 2012, ApJL, 757, L29

Allers, K. N., Kessler-Silacci, J. E., Cieza, L. A., \& Jaffe, D. T. 2006, ApJ, 644,364

Andrews, S., \& Williams, J. 2007a, ApJ, 671, 1800

Andrews, S. M., \& Williams, J. P. 2007b, ApJ, 671, 1800

Andrews, S. M., Wilner, D. J., Espaillat, C, et al. 2011, ApJ, 732, 25pp

Andrews, S. M., Wilner, D. J., Hughes, A. M., Qi, C., \& Dullemond, C. P. 2009, ApJ, 700, 1502

Artymowicz, \& Lubow 1994, ApJ, 421, 651

Baraffe, I., \& Chabrier, G. 2010, A\&A, 521, A44

Baraffe, I., Chabrier, G., Allard, F., \& Hauschildt, P. H. 1998, A\&A, 337,403

Baraffe, I., Chabrier, G., \& Gallardo, J. 2009, ApJL, 702, L27

Baraffe, I., Homeier, D., Allard, F., \& Chabrier, G. 2015, A\&A, 577, A42

Baraffe, I., Vorobyov, E., \& Chabrier, G. 2012, ApJ, 756, 118

Barsony, M., Ressler, M. E., \& Marsh, K. A. 2005, ApJ, 630, 381

Beichman, C. A., Neugebauer, G., Habing, H. J., Clegg, P. E., \& Chester, T. J. (ed.) 1988, Infrared Astronomical Satellite (IRAS) Catalogs and Atlases, Vol. 1 (Washington, D. C.: NASA)

Berdyugina, S. V. 2005, LRSP, 2, 8

Bernstein, R., Shectman, S. A., Gunnels, S. M., Mochnacki, S., \& Athey, A. E. 2003, Proc. SPIE, 4841, 1694

Beuther, H., Klessen, R., Dullemond, C., \& Henning, T. 2014, Protostars and Planets VI (Tucson, AZ: Univ. Arizona Press)

Biller, B., Lacour, S., Juhász, A., et al. 2012, ApJL, 753, L38

Bontemps, S., André, P., Kaas, A. A., et al. 2001, A\&A, 372, 173

Brown, J. M., Blake, G. A., Qi, C., et al. 2009, ApJ, 704, 496

Burki, G., \& Mayor, M. 1986, in IAU Symp. 118, Instrumentation and Research Programmes for Small Telescopes, ed. J. B. Hearnshaw, \& P. L. Cottrell (Dordrecht: Riedel), 385

Carroll, J. A. 1933, MNRAS, 93, 478

Casagrande, L., Flynn, C., \& Bessell, M. 2008, MNRAS, 389, 585

Chabrier, G., Baraffe, I., \& Plez, B. 1996, ApJL, 459, L91

Cheetham, A. C., Kraus, A. L., Ireland, M. J., et al. 2015, ApJ, 813, 83

Cieza, L., Padgett, D. L., Allen, L. E., et al. 2009, ApJL, 696, L84

Cieza, L., Padgett, D. L., Stapelfeldt, K. R., et al. 2007, ApJ, 667, 308

Cieza, L. A., Schreiber, M. R., Romero, G. A., et al. 2010, ApJ, 712, 925

Claret, A. 2000, A\&A, 363, 1081

Cruz, K. L., \& Reid, I. N. 2002, AJ, 123, 2828

Cutri, R. M., Skrutskie, M. F., van Dyk, S., et al. 2003, 2MASS All Sky Catalog of Point Sources

Daemgen, S., Siegler, N., Reid, I. N., \& Close, L. M. 2007, AJ, 654, 558

D’Alessio, P., Merin, B., Calvet, N., Hartmann, L., \& Montesinos, B. 2005, RMxAA, 41, 61

Desort, M., Lagrange, A.-M., Galland, F., Udry, S., \& Mayor, M. 2007, A\&A, 473, 983

Dodson-Robinson, S. E., \& Salyk, C. 2011, ApJ, 738, 131

Doyle, L. R., Carter, J. A., Fabrycky, D. C., et al. 2011, Sci, 333, 1602

Ducati, J. R. 2002, yCat, 2237, 0

Duchêne, G. 2010, ApJL, 709, L114

Duncan, D. K. 1981, ApJ, 248, 651

Duquennoy, A., \& Mayor, M. 1991, A\&A, 248, 485
Eisner, J. A., Monnier, J. D., Tuthill, P., \& Lacour, S. 2009, ApJL, 698, L169 Erickson, K. L., Wilking, B. A., Meyer, M. R., Robinson, J. G., \& Stephenson, L. N. 2011, AJ, 142, 140

Espaillat, C., Ingleby, L., Hernández, J., et al. 2012, ApJ, 747, 103

Evans, N. J., II, Allen, L. E., Blake, G. A., et al. 2003, PASP, 115, 965

Evans, N. J., II, Dunham, M. M., Jørgensen, J. K., et al. 2009, ApJS, 181, 321

Feigelson, E. D., \& Lawson, W. A. 2004, ApJ, 614, 267

Fischer, D., \& Marcy, G. 1992, ApJ, 396, 178

Fitzpatrick, M. J. 1993, in ASP Conf. Ser. 52, Astronomical Data Analysis Software and Systems II, ed. R. J. Hanisch, R. J. V. Brissenden, \& J. Barnes (San Francisco, CA: ASP), 472

Follette, K. B., Tamura, M., Hashimoto, J., et al. 2013, ApJ, 767, 10

Forrest, W. J., Sargent, B., Furlan, E., et al. 2004, ApJS, 154, 443

Furlan, E., Watson, D. M., McClure, M. K., et al. 2009, ApJ, 703, 1964

Geers, V. C., van Dishoeck, E. F., Visser, R., et al. 2007, A\&A, 476, 279

Ghez, A. M., McCarthy, D. W., Patience, J. L., \& Beck, T. L. 1997, ApJ, 481,378

Ghez, A. M., Neugebauer, G., \& Matthews, K. 1993, AJ, 106, 2005

Gizis, J. E. 1997, AJ, 113, 806

Gorti, U., Dullemond, C. P., \& Hollenbach, D. 2009, ApJ, 705, 1237

Gray, D. F. 1976, The Observation and Analysis of Stellar Photospheres (New York: Wiley Interscience)

Gray, D. F. 2005, The Observation and Analysis of Stellar Photospheres (Cambridge: Cambridge Univ. Press)

Hamilton, C. M., Johns-Krull, C. M., Mundt, R., Herbst, W., \& Winn, J. N. 2012, ApJ, 751, 147

Hatzes, A. 2002, AN, 323, 392

Hilditch, R. W. 2001, An Introduction to Close Binary Stars (Cambridge: Cambridge Univ. Press)

Hughes, A. M., Andrews, S. M., Espaillat, C., et al. 2009, ApJ, 698, 131

Hughes, A. M., Andrews, S. M., Wilner, D. J., et al. 2010, AJ, 140, 887

Hughes, A. M., Wilner, D. J., Calvet, N., et al. 2007, ApJ, 664, 536

Ireland, M. J., \& Kraus, A. L. 2008, ApJL, 678, L59

James, D. J., Melo, C., Santos, N. C., \& Bouvier, J. 2006, A\&A, 446, 971

Jayawardhana, R., Mohanty, S., \& Basri, G. 2003, ApJ, 592, 282

Johns-Krull, C. M., \& Valenti, J. A. 2001, ApJ, 561, 1060

Kelson, D. 2003, PASP, 115, 688

Kim, K. H., Watson, D. M., Manoj, P., et al. 2009, ApJ, 700, 1017

Kimeswenger, S., Lederle, C., Richichi, A., et al. 2004, A\&A, 413, 1037

Kiraga, M. 2012, AcA, 62, 67

Knacke, R. F., Strom, K. M., Strom, S. E., Young, E., \& Kunkel, W. 1973, ApJ, 179, 847

Kraus, A. L., Ireland, M. J., Hillenbrand, L. A., \& Martinache, F. 2012, ApJ, 745,19

Kurosawa, R., Harries, T. J., \& Littlefair, S. P. 2006, MNRAS, 372, 1879

Lafreniere, D., Jayawardhana, R., Brandeker, A., Ahmic, M., \& van Kerkwijk, M. H. 2008, ApJ, 683, 844

Lodieu, N., Pérez-Garrido, A., Béjar, V. J. S., et al. 2014, A\&A, 569, A120

Lombardi, M., Lada, C. J., \& Alves, J. 2008, A\&A, 480, 785

Lopez Martí, B., Jimenez Esteban, F., Bayo, A., et al. 2013, A\&A, 551, A46

Magazzu, A., Rebolo, R., \& Pavlenko, I. V. 1992, ApJ, 392, 159

Mamajek, E. E. 2009, in AIP Con. Ser. 1158, ed. T. Usuda, M. Tamura, \& M. Ishii (Melville, NY: AIP), 3

Marcy, G. W., \& Benitz, K. J. 1989, ApJ, 344, 441 
Martin, E. L., Basri, G., \& Zapatero Osorio, M. R. 1999a, AJ, 118, 1005

Martin, E. L., Delfosse, X., Basri, G., et al. 1999b, AJ, 118, 2466

Melo, C. H. F. 2003, A\&A, 410, 269

Mohanty, S., Basri, G., Shu, F., Allard, F., \& Chabrier, G. 2002, ApJ, 571, 469

Monet, D. G., Levine, S. E., Canzian, B., et al. 2003, ApJ, 125, 984

Muzerolle, J., Allen, L. E., Megeath, S. T., Hernández, J., \& Gutermuth, R. A. 2010, ApJ, 708, 1107

Natta, A., Testi, L., Muzerolle, J., et al. 2004, A\&A, 424, 603

Neugebauer, G., Habing, H. J., van Duinen, R., et al. 1984, ApJL, 278, L1

Neuhauser, R., \& Forbrich, J. 2008, in Handbook of Star Forming Regions, The Southern Sky, Vol. II, Vol. 5, ed. B. Reipurth (San Francisco, CA: ASP), 735

Neuhäuser, R., Walter, F. M., Covino, E., et al. 2000, A\&AS, 146, 323

Nguyen, D. C., Scholz, A., van Kerkwijk, M. H., Jayawardhana, R., \& Brandeker, A. 2009, ApJL, 694, L153

Nidever, D. L., Marcy, G. W., Butler, R. P., Fischer, D. A., \& Vogt, S. S. 2002, ApJS, 141, 503

Orosz, J. A., Welsh, W. F., Carter, J. A., et al. 2012, Sci, 337, 1511

Padgett, D. L., Rebull, L. M., Stapelfeldt, K. R., et al. 2008, ApJ, 672, 1013

Pinilla, P., Benisty, M., \& Birnstiel, T. 2012, A\&A, 545, A81

Pott, J.-U., Perrin, M. D., Furlan, E., et al. 2010, ApJ, 710, 265

Prato, L. 2007, ApJ, 657, 338

Prato, L., Greene, T. P., \& Simon, M. 2003, ApJ, 584, 853

Qian, S.-B., Liu, L., Zhu, L.-Y., et al. 2012, MNRAS, 422, 24

Qian, S.-B., Zhu, L.-Y., Dai, Z.-B., et al. 2012b, ApJL, 745, L23

Quillen, A. C., Blackman, E. G., Frank, A,.. \& Varniere, P. 2004, ApJL, 612, L137

Raghavan, D., McAlister, H. A., Henry, T. J., et al. 2010, ApJS, 190, 1

Rajpurohit, A. S., Reylé, C., Allard, F., et al. 2013, A\&A, 556, A15

Ratzka, T., Kohler, R., \& Leinert, C. 2005, A\&A, 437, 611

Reid, I. N., \& Cruz, K. L. 2002, AJ, 123, 2806

Reiners, A., Schmitt, J. H. M. M., \& Kürster, M. 2001, A\&A, 376, L13

Rice, W. K. M., Armitage, P. J., Wood, K., \& Lodato, G. 2006, MNRAS, 373,1619
Richichi, A., \& Percheron, I. 2002, A\&A, 386, 492

Richichi, A., Percheron, I., \& Khristoforova, M. 2005, A\&A, 431, 773

Rosero, V., Prato, L., Wasserman, L. H., \& Rodgers, B. 2011, AJ, 141, 13

Samus, N. N., Goranskii, V. P., Durlevich, O. V., et al. 2003, AstL 29,468

Schwamb, M. E., Orosz, J. A., Carter, J. A., et al. 2013, ApJ, 768, 127

Shkolnik, E., Liu, M. C., \& Reid, I. N. 2009, ApJ, 699, 649

Shkolnik, E. L., Hebb, L., Liu, M. C., Reid, I. N., \& Collier Cameron, A. 2010, ApJ, 716, 1522

Sicilia-Aguilar, A., Hartmann, L., Calvet, N., et al. 2006, ApJ, 638, 897

Sicilia-Aguilar, A., Henning, T., Juhasz, A., et al. 2008, ApJ, 687, 1145

Siess, L., Dufour, E., \& Forestini, M. 2000, A\&A, 358, 593

Simón-Díaz, S., \& Herrero, A. 2007, A\&A, 468, 1063

Skrutskie, M. F., Cutri, R. M., Stiening, R., et al. 2006, AJ, 131, 1163

Struve, O., \& Rudkjobing, M. 1949, ApJ, 109, 92

Suarez, O., Garcia-Lario, P., Manchado, A., et al. 2006, A\&A, 458, 173

Torres, C. A. O., Quast, G. R., da Silva, L., et al. 2006, A\&A, 460, 695

Vogt, N., Schmidt, T. O. B., Neuhäuser, R., et al. 2012, A\&A, 546, A63

Vrba, F. J., Coyne, G. V., \& Tapia, S. 1993, AJ, 105, 1010

Wahhaj, Z., Cieza, L., Koerner, D. W., et al. 2010, ApJ, 724, 835

White, R. J., \& Basri, G. 2003, ApJ, 582, 1109

White, R. J., Gabor, J. M., \& Hillenbrand, L. A. 2007, AJ, 133, 2524

White, R. J., \& Ghez, A. M. 2001, ApJ, 556, 265

White, R. J., \& Hillenbrand, L. A. 2005, ApJL, 621, L65

Wilking, B. A., Meyer, M. R., Robinson, J. G., \& Greene, T. P. 2005, AJ, 130,1733

Wyatt, W. F. 1985, in Stellar Radial Velocities, ed. A. G. D. Philip, \& D. W. Latham (Schenectady: L Davis Press), 123

Zhu, Z., Nelson, R. P., Hartmann, L., Espaillat, C., \& Calvet, N. 2011, ApJ, 729,47

Zhu, Z., \& Stone, J. M. 2014, ApJ, 795, 53

Zickgraf, F.-J., Krautter, J., Reffert, S., et al. 2005, A\&A, 433, 151

Zuckerman, B., \& Song, I. 2004, ARA\&A, 42, 685 